\title{
Alternative Frequency and Time Domain Versions of Fractional Brownian Motion
}

\author{
James Davidson* and Nigar Hashimzade \\ University of Exeter \\ March 2006 \\ Forthcoming in Econometric Theory.
}

\begin{abstract}
This paper compares models of fractional processes and associated weak convergence results based on moving average representations in the time domain with spectral representations. Both approaches have been applied in the literature on fractional processes. We point out that the conventional forms of these models are not equivalent, as is commonly assumed, even under a Gaussianity assumption. We show that it is necessary to distinguish between 'two-sided' processes depending on both leads and lags from one-sided or 'causal' processes, since in the case of fractional processes these models yield different limiting properties. We derive new representations of fractional Brownian motion, and show how different results are obtained for, in particular, the distribution of stochastic integrals in the multivariate context. Our results have implications for valid statistical inference in fractional integration and cointegration models.
\end{abstract}

\footnotetext{
${ }^{*}$ Corresponding author, james.davidson@exeter.ac.uk. We thank F. Hashimzade and two anonymous referees for their valuable comments.
} 


\section{Introduction}

Two approaches to studying the asymptotics of long memory, fractional integration and cointegration models have been developed in recent literature. In the first of these, which we call the 'time domain moving average' model, the fractionally integrated process is commonly written as

$$
x_{t}=(1-L)^{-d} u_{t}
$$

where $L$ represents the lag operator, $|d|<\frac{1}{2}$, and $u_{t}$ is a short memory (weakly dependent) process. As is well known, the binomial expansion of $(1-L)^{-d}$ yields the infinite moving average representation

$$
x_{t}=\sum_{j=0}^{\infty} b_{j} u_{t-j}
$$

where

$$
b_{j}=\frac{\Gamma(d+j)}{\Gamma(d) \Gamma(j+1)} .
$$

and $\Gamma()$ denotes the gamma function. Under the indicated restriction on $d$ the lag coefficients are square summable, and the process is accordingly covariance stationary. In many applications we consider the linear case where

$$
u_{t}=\theta(L) \varepsilon_{t}
$$

with $\left\{\varepsilon_{t}\right\}$ i.i.d. $\left(0, \sigma^{2}\right)$ and $\theta(L)=\sum_{l=0}^{\infty} \theta_{j} L^{j}$, having absolutely summable coefficients. See, for example, Granger and Joyeux (1980), Hosking (1981), Beran (1994) and many related references.

In the second approach, the frequency domain harmonizable representation of the process is adopted. Define

$$
x_{t}=\int_{-\pi}^{\pi} e^{i t \lambda} h\left(e^{i \lambda}\right) W(d \lambda)
$$

where $i$ is the imaginary unit and $W$ is a complex-valued Gaussian random measure with the properties

$$
\begin{aligned}
W(-d \lambda) & =\overline{W(d \lambda)} \\
E(W(d \lambda)) & =0 \\
E(W(d \lambda) \overline{W(d \mu)}) & =\left\{\begin{array}{cc}
\sigma^{2} d \lambda, & \mu=\lambda \\
0, & \text { otherwise }
\end{array}\right.
\end{aligned}
$$

Observe that $x_{t}$ is always real-valued since the integrals over $[-\pi, 0]$ and $[0, \pi]$ are complex conjugates. $h\left(e^{i \lambda}\right)$ is called the transfer function or frequency response function of the process. If $x_{t}$ is i.i.d. then $h\left(e^{i \lambda}\right)=1 / \sqrt{2 \pi}$ (constant), but otherwise we wish to think of it at this stage merely as some function of $\lambda$, complex-valued in general, to be specified by the context. It is however related to the spectral density of the process by $f(\lambda)=\sigma^{2}\left|h\left(e^{i \lambda}\right)\right|^{2}$, and in the long memory case the latter function is often represented in the generic form

$$
f(\lambda)=|\lambda|^{-2 d} L(\lambda)
$$

where $d$ matches the parameter in (1.1), and $L(\lambda)$ is at most slowly varying at $0 .{ }^{1}$ See Robinson (1994b), Brockwell and Davis (1991) Section 13.2, among other references, for further details of this type of model. Note that the harmonizable representation is more specialized than the time

\footnotetext{
${ }^{1}$ Here, $L()$ is the standard generic notation for a slowly varying function, not to be confused with the lag operator $L$.
} 
domain model since it requires the process to be Gaussian and stationary, whereas $u_{t}$ in (1.1) can belong to a more general class of processes. However, these models are often treated, explicitly or implicitly, as being merely alternative representations of the same process. A key feature of the theory is that the associated normalized partial-sum processes should converge weakly to a Gaussian a.s.-continuous limit process known as fractional Brownian motion (fBM).

The object of this paper is to show that there are, in fact, important differences between these models as commonly represented, and that, in particular, alternative versions of the fractional model lead to different limit processes. The differences are not trivial, and may have completely different implications for joint distributions in the multivariate case. Section 2 describes a class of harmonizable representations of fractional Brownian motion, all sharing a common spectrum apart from scale constants, and shows how they can be derived as the weak limits of forward and/or backward-looking linear moving average processes. Section 3 considers the multivariate case, and examines the cross-spectra and covariance functions of different members of the class. Section 4 considers the distribution of the stochastic integral of one process with respect to the increments of another, and generalizations of Chan and Terrin's (1995) weak convergence results are proved. Section 5 presents a complementary analysis of time domain representations, generalizing the model first proposed by Mandelbrot and van Ness (1968), and gives a generalized weak convergence result for this class using the approach of Davidson and de Jong (2000). Finally, Section 6 discusses our results in the context of the existing literature, and shows how they resolve a number of puzzles and contradictory features of this literature. Proofs are gathered in the Appendix.

\section{Harmonizable Representations of Fractional Brownian Motion}

In this section we introduce a class of continuous time Gaussian processes,

$$
X(r ; d, \kappa, a)=\frac{1}{\sqrt{2 \pi}} \int_{-\infty}^{\infty} \frac{e^{i \lambda r}-1}{i \lambda} \tilde{g}(\lambda ; d, \kappa, a) W(d \lambda)
$$

where $W$ is a complex-valued Gaussian process on $\mathbb{R}$ satisfying the conditions of (1.3), and $\tilde{g}$ is a complex valued transfer function having the general form

$$
\tilde{g}(\lambda ; d, \kappa, a)=\kappa(i \lambda)^{a-d}(-i \lambda)^{-a}+(1-\kappa)(i \lambda)^{-a}(-i \lambda)^{a-d} .
$$

for real constants $d, \kappa, a$, where $-\frac{1}{2} \leq d<\frac{1}{2}, 0 \leq a \leq d / 2$ for $d>0$ and $d / 2 \leq a \leq 0$ for $d<0$, and $0 \leq \kappa \leq 1$.

We mention two points of clarification at the outset. The first is to emphasize that this model class is introduced as a vehicle for developing and illustrating the representation issues addressed in the paper. We do not attempt to assign any particular interpretation to the parameters $\kappa$ and $a$ which, as we show, can be thought of as indexing different distributed lead/lag structures. We will, in fact, argue that most practical applications in econometrics are covered by one particular case (see (2.8) below). Second, note that in the sequel we use the generic notation $X(r)$ to denote any member of the class, so that dependence on the three parameters is taken as implicit. This is chiefly for notational clarity, since the expressions involving several coordinates of $X$ would otherwise become unwieldy.

Now, note the identity

$$
(\mp i \lambda)^{s}=(\mp i \operatorname{sgn}(\lambda))^{s}|\lambda|^{s}=\exp \left(\mp i \operatorname{sgn}(\lambda) s \frac{\pi}{2}\right)|\lambda|^{s}
$$

where $\operatorname{sgn}(\lambda)=1$ if $\lambda \geq 0$ and -1 otherwise. Thus, (2.2) can also be written as

$$
\tilde{g}(\lambda ; d, \kappa, a)=|\lambda|^{-d} \Upsilon(\lambda ; d, \kappa, a)
$$


where

$$
\Upsilon(\lambda ; d, \kappa, a)=\kappa \exp (-i \pi(d / 2-a) \operatorname{sgn}(\lambda))+(1-\kappa) \exp (i \pi(d / 2-a) \operatorname{sgn}(\lambda)) .
$$

Further note that

$$
\begin{aligned}
|\Upsilon(\lambda ; d, \kappa, a)|^{2} & =1-4 \kappa(1-\kappa) \sin ^{2} \pi\left(\frac{d}{2}-a\right) \\
& =K(d, \kappa, a)^{2}
\end{aligned}
$$

(defining $K(d, \kappa, a))$ and so the generalized spectral densities of this class of processes take the form

$$
|\tilde{g}(\lambda ; d, \kappa, a)|^{2}=|\lambda|^{-2 d} K(d, \kappa, a)^{2} .
$$

Alternatively, the variance of a process increment of width $\delta>0$, for any $r \in[0,1-\delta]$, is

$$
\begin{aligned}
E(X(r+\delta) & -X(r))^{2} \\
& =\frac{1}{2 \pi} E \int_{-\infty}^{\infty} \frac{e^{i \lambda(r+\delta)}-e^{i \lambda r}}{i \lambda} \tilde{g}(\lambda ; d, \kappa, a) W(d \lambda) \\
& \times \int_{-\infty}^{\infty} \frac{e^{i \mu(r+\delta)}-e^{i \mu r}}{i \mu} \tilde{g}(\mu ; d, \kappa, a) W(d \mu) \\
& =\frac{\sigma^{2}}{2 \pi} K(d, \kappa, a)^{2} 2 \int_{-\infty}^{\infty}(1-\cos \lambda \delta)|\lambda|^{-2 d-2} d \lambda \\
& =\frac{\sigma^{2} K(d, \kappa, a)^{2}}{\Gamma(2 d+2) \cos \pi d} \delta^{2 d+1}
\end{aligned}
$$

where the last equality can be verified from Gradshteyn and Ryzhik (2000) (henceforth GR) Relation 3.823. Applying the identity

$$
\begin{gathered}
\left(X\left(r_{4}\right)-X\left(r_{3}\right)\right)\left(X\left(r_{2}\right)-X\left(r_{1}\right)\right)=\frac{1}{2}\left[\left(X\left(r_{4}\right)-X\left(r_{1}\right)\right)^{2}+\left(X\left(r_{3}\right)-X\left(r_{2}\right)\right)^{2}\right. \\
\left.-\left(X\left(r_{3}\right)-X\left(r_{1}\right)\right)^{2}-\left(X\left(r_{4}\right)-X\left(r_{2}\right)\right)^{2}\right] .
\end{gathered}
$$

for any $r_{1}, r_{2}, r_{3}, r_{4} \in[0,1]$ with $r_{1}<r_{2}$ and $r_{3}<r_{4}$, formula (2.6) can be used to derive the covariance of any pair of segments of the process. The covariance structure of these processes therefore depends on $a$ and $\kappa$ only through the scale factor $K(d, \kappa, a)$, and otherwise matches that of what is usually called 'fractional Brownian motion'. Since in general $\sigma^{2}$ is unknown, these parameters are accordingly unidentified from the point of view of a single process.

Any of these processes are candidates to be referred to as fractional Brownian motion, and some leading examples of the class have been proposed in the literature. Setting $\kappa=1$ and $a=0$ yields

$$
X(r)=\frac{1}{\sqrt{2 \pi}} \int_{-\infty}^{\infty} \frac{e^{i \lambda r}-1}{i \lambda}(i \lambda)^{-d} W(d \lambda)
$$

(See e.g. Reed, Lee and Truong 1995). Setting $a=d / 2$ on the other hand (in which case $\kappa$ drops out) yields

$$
X(r)=\frac{1}{\sqrt{2 \pi}} \int_{-\infty}^{\infty} \frac{e^{i \lambda r}-1}{i \lambda}|\lambda|^{-d} W(d \lambda)
$$

which, apart from an at most slowly varying component, is the model examined extensively by Chan and Terrin (1995). As a further variant, set $\kappa=\frac{1}{2}$ and $a=0$ to yield

$$
X(r)=\frac{1}{\sqrt{2 \pi}} \int_{-\infty}^{\infty} \frac{e^{i \lambda r}-1}{i \lambda} \operatorname{Re}(i \lambda)^{-d} W(d \lambda) .
$$


The last case has not been proposed to date, to our knowledge, but provides an alternative to (2.9) as a way of representing symmetry of the backward and forward-looking dependence. As we show, this property is implicit in any model having a real-valued transfer function.

As noted, Chan and Terrin (1995), in common with other contributions to this literature, allow slowly varying components in the transfer function and spectral density. Our class of models might easily be elaborated to include, for example, a slowly varying, even function of $\lambda$ as a factor in (2.3). Such extensions would complicate the analysis, but would not change the basic conclusions. While these issues would be of interest to explore further, we choose to focus the present discussion on the model as given.

One way to to motivate our class is by considering a corresponding class of discrete processes in the time domain. We may then show how the Brownian motions derived above can be viewed as weak limits as the time intervals shrink to zero. Let

$$
u_{t}=\frac{1}{\sqrt{2 \pi}} \int_{-\pi}^{\pi} e^{i \lambda t} W(d \lambda)
$$

represent a serially independent Gaussian process with mean 0 and variance $\sigma^{2}$, and so consider a 'two-sided' moving average process

$$
x_{t}=g(L ; d, \kappa, a) u_{t}=\sum_{j=-\infty}^{\infty} b_{j} u_{t-j}
$$

where

$$
g(L ; d, \kappa, a)=\kappa(1-L)^{a-d}\left(1-L^{-1}\right)^{-a}+(1-\kappa)(1-L)^{-a}\left(1-L^{-1}\right)^{a-d} .
$$

It is easily verified that the lag structure is a convolution of the binomial series associated with fractional integration. Thus,

$$
\begin{aligned}
b_{0} & =\frac{1}{\Gamma(a) \Gamma(d-a)} \sum_{k=0}^{\infty} \frac{\Gamma(a+k) \Gamma(d-a+k)}{\Gamma(k+1)^{2}}, \\
b_{j} & =\frac{1}{\Gamma(a) \Gamma(d-a)} \sum_{k=0}^{\infty}\left(\frac{\kappa \Gamma(a+k) \Gamma(d-a+k+j)+(1-\kappa) \Gamma(d-a+k) \Gamma(a+k+j)}{\Gamma(k+1) \Gamma(k+j+1)}\right), \\
b_{-j} & =\frac{1}{\Gamma(d-a) \Gamma(a)} \sum_{k=0}^{\infty}\left(\frac{\kappa \Gamma(d-a+k) \Gamma(a+k+j)+(1-\kappa) \Gamma(a+k) \Gamma(d-a+k+j)}{\Gamma(k+1) \Gamma(k+j+1)}\right)
\end{aligned}
$$

for $j>0$. Note that

$$
\begin{aligned}
\sum_{k=0}^{\infty} \frac{\Gamma(a+k) \Gamma(d-a+k+j)}{\Gamma(k+1) \Gamma(k+j+1)} & \sim \sum_{k=0}^{\infty} k^{a-1}(j+k)^{d-a-1} \\
& =j^{d-2} \sum_{k=0}^{j}\left(\frac{k}{j}\right)^{a-1}\left(1+\frac{k}{j}\right)^{d-a-1}+\sum_{k=j+1}^{\infty} k^{d-2}\left(\frac{j}{k}+1\right)^{d-a-1} \\
& \sim A j^{d-1}
\end{aligned}
$$

for $0<A<\infty$, and similarly

$$
\sum_{k=0}^{\infty} \frac{\Gamma(d-a+k) \Gamma(a+k+j)}{\Gamma(k+1) \Gamma(k+j+1)} \sim \sum_{k=0}^{\infty} k^{d-a-1}(j+k)^{a-1} \sim A j^{d-1} .
$$


Hence, with $d>0$ this process has long memory with parameter $d$ 'in the tails' of the lag/lead distributions. However, different choices of $a$ and $\kappa$, given $d$, influence the amount of short-run memory relative to long run, either symmetrically forwards and backwards or asymmetrically.

The case $a=0, \kappa=1$ yields $g(L)=(1-L)^{-d}$. This, of course, corresponds uniquely to the one-sided (causal) model, with $b_{j}=0$ for $j>0$ (lags but no leads). With $a=0, \kappa=0$, we have $g(L)=\left(1-L^{-1}\right)^{-d}$ (leads but no lags). On the other hand, with $a=d / 2$ the model does not depend on $\kappa$ and $g(L)=\left(2-L-L^{-1}\right)^{-d / 2}$, so that $b_{j}=b_{-j}$ for each $j>0$. The symmetry property also holds for the case $\kappa=\frac{1}{2}$. Otherwise, with $a$ lying strictly between zero and $d / 2$, the models exhibit different degrees of forward and backward memory.

On substituting the harmonizable representation of $u_{t}$ into $(2.11)$, we obtain ${ }^{2}$

$$
\begin{aligned}
x_{t} & =\frac{1}{\sqrt{2 \pi}} \int_{-\pi}^{\pi} e^{i \lambda t} \sum_{j=-\infty}^{\infty} b_{j} e^{-i \lambda j} W(d \lambda) \\
& =\frac{1}{\sqrt{2 \pi}} \int_{-\pi}^{\pi} e^{i \lambda t} g\left(e^{-i \lambda} ; d, \kappa, a\right) W(d \lambda) .
\end{aligned}
$$

Introducing a change of variable from $\lambda$ to $\lambda / n$, consider the partial sum process

$$
X_{n}(r)=n^{-1 / 2-d} \sum_{t=1}^{[n r]} x_{t}
$$

and note that

$$
\begin{aligned}
X_{n}(r) & =\frac{1}{n^{d+1} \sqrt{2 \pi / n}} \int_{-\pi}^{\pi} \sum_{t=1}^{[n r]} e^{i \lambda t} g\left(e^{-i \lambda} ; d, \kappa, a\right) W(d \lambda) \\
& =\frac{1}{\sqrt{2 \pi}} \int_{-n \pi}^{n \pi} \frac{e^{i \lambda([n r]+1) / n}-e^{i \lambda / n}}{n\left(e^{i \lambda / n}-1\right)} \frac{g\left(e^{-i \lambda / n} ; d, \kappa, a\right)}{n^{d}} W(d \lambda) \\
& \approx \frac{1}{\sqrt{2 \pi}} \int_{-n \pi}^{n \pi} \frac{e^{i \lambda r}-1}{i \lambda} \tilde{g}(\lambda ; d, \kappa, a) W(d \lambda)
\end{aligned}
$$

where $" \approx "$ means that the approximation improves as $n$ increases. Following the first part of Chan and Terrin (1995)'s Theorem 3.3 we can formalize this intuition as follows, where $X$ is defined by (2.1).

Theorem $2.1 X_{n} \stackrel{d}{\rightarrow} X$ for any $0 \leq d<\frac{1}{2}, 0 \leq \kappa \leq 1$ and $0 \leq a \leq d / 2$.

Note that, unlike Chan and Terrin's theorem, this result covers the standard Brownian motion case $d=0$. It still does not cover the case of $-\frac{1}{2}<d<0$, for which weak convergence is a wellestablished property of the time domain representation (see Davidson and de Jong 2000, inter alia). It turns out that our proof does not work for this case, because the integral in the majorant side of (A-1) is non-convergent. However, we also note that where the discrete-time process is both linear and Gaussian, since uniform tightness holds it is sufficient, in view of the identities in (2.7) and (3.7), to establish that the limit of the variances of the partial sum process matches that of $X$. Accordingly, we can prove the following:

\footnotetext{
${ }^{2}$ Observe that in the symmetric example $a=d / 2$ just noted, the transfer function $g\left(e^{-i \lambda}\right)=\left(2-e^{-i \lambda}-\right.$ $\left.e^{i \lambda}\right)^{-d / 2}=(2-2 \cos \lambda)^{-d / 2}$ is simply the square root of the spectral density.
} 
Theorem 2.2 If

$$
x_{t}=\frac{1}{\sqrt{2 \pi}} \int_{-\pi}^{\pi} e^{i \lambda t}\left(1-e^{-i \lambda}\right)^{-d} W(d \lambda)
$$

for $-\frac{1}{2}<d<\frac{1}{2}$, and $X_{n}(r)$ is defined by (2.13), then $X_{n} \stackrel{d}{\rightarrow} X$ where $X(r)$ is defined by (2.8).

While this statement of the result is given only for the causal member of our class having $\kappa=1$ and $a=0$, note that both the finite- $n$ and limiting variance expressions are generalized to the cases with $0 \leq \kappa \leq 1$ and $d / 2 \leq a \leq 0$ simply by applying the factor $K(d, \kappa, a)^{2}$. The result may be easily extended in this way to our complete class of Gaussian linear processes.

\section{The Multivariate Case}

It is clear that all processes $x_{t}$ defined by (2.11) have the same spectral density function, and accordingly the limiting partial sum processes have the same autocovariance structure, the one usually identified with 'fractional Brownian motion'. However, it is also the case that these processes are distinct, and we now consider how they might be distinguished. This can only be in terms of the relations between different processes.

Consider processes $X_{1}$ and $X_{2}$ defined as in (2.1) with parameters $\left(d_{1}, \kappa_{1}, a_{1}\right)$ and $\left(d_{2}, \kappa_{2}, a_{2}\right)$ and driving processes $W_{1}, W_{2}$. The latter are complex-valued Gaussian random measures with the properties (for $j, k=1,2$ )

$$
\begin{aligned}
W_{j}(-d \lambda) & =\overline{W_{j}(d \lambda)} \\
E W_{j}(d \lambda) & =0 \\
E W_{j}(d \lambda) \overline{W_{k}(d \mu)} & =\left\{\begin{array}{cc}
\sigma_{j k} d \lambda, & \mu=\lambda \\
0, & \text { otherwise. }
\end{array}\right.
\end{aligned}
$$

In this section and the one following we let $0 \leq d_{j}<\frac{1}{2}$ for $j=1,2$ We do not attempt to treat the cases $d_{j}<0$ explicitly, although we conjecture that these extensions might be developed on the lines of Theorem 2.2 .

Define

$$
\begin{aligned}
P_{12}(\lambda) & =\Upsilon\left(\lambda ; d_{1}, \kappa_{1}, a_{1}\right) \Upsilon\left(-\lambda ; d_{2}, \kappa_{2}, a_{2}\right) . \\
& =\sum_{j=1}^{4} \theta_{j} \exp \left(i \pi \xi_{j} \operatorname{sgn}(\lambda)\right)
\end{aligned}
$$

where

$$
\begin{array}{ll}
\theta_{1}=\kappa_{1} \kappa_{2}, & \xi_{1}=-\left(a_{1}-a_{2}-\frac{d_{1}-d_{2}}{2}\right) \\
\theta_{2}=\left(1-\kappa_{1}\right)\left(1-\kappa_{2}\right), & \xi_{2}=a_{1}-a_{2}-\frac{d_{1}-d_{2}}{2} \\
\theta_{3}=\kappa_{1}\left(1-\kappa_{2}\right), & \xi_{3}=-\left(a_{1}+a_{2}-\frac{d_{1}+d_{2}}{2}\right) \\
\theta_{4}=\left(1-\kappa_{1}\right) \kappa_{2} & \xi_{4}=a_{1}+a_{2}-\frac{d_{1}+d_{2}}{2}
\end{array}
$$

Observe, from (2.1), that the cross-spectrum of the increments is defined as

$$
\begin{aligned}
f_{12}(\lambda) & =\tilde{g}(\lambda ; d, \kappa, a) \tilde{g}\left(-\lambda ; d_{2}, \kappa_{2}, a_{2}\right) \\
& =|\lambda|^{-d_{1}-d_{2}} P_{12}(\lambda) .
\end{aligned}
$$


We can notice, for example, that $f_{12}(\lambda)$ is real-valued in the 'time symmetric' cases, where either $a_{1}=d_{1} / 2$ and $a_{2}=d_{2} / 2$, or $\kappa_{1}=\kappa_{2}=\frac{1}{2}$, although not in general. However, we can potentially learn more by considering time-domain covariances of the Brownian motions, extending the formulation in (2.6).

Consider the case of contemporaneous increments.

Proposition 3.1 For $\delta>0$ and $0 \leq r \leq 1-\delta$,

$$
\begin{aligned}
& E\left[\left(X_{2}(r+\delta)-X_{2}(r)\right)\left(X_{1}(r+\delta)-X_{1}(r)\right)\right] \\
& =\frac{\sigma_{12} \delta^{d_{1}+d_{2}+1}}{\Gamma\left(2+d_{1}+d_{2}\right) \cos \pi \frac{d_{1}+d_{2}}{2}}\left[\cos \pi\left(a_{1}-a_{2}-\frac{d_{1}-d_{2}}{2}\right)-\right. \\
& \left.\quad 2\left[\kappa_{1}\left(1-\kappa_{2}\right)+\left(1-\kappa_{1}\right) \kappa_{2}\right] \sin \pi\left(a_{1}-\frac{d_{1}}{2}\right) \sin \pi\left(a_{2}-\frac{d_{2}}{2}\right)\right]
\end{aligned}
$$

Note that this formula reduces to (2.6) in the case $X_{1}=X_{2}=X$. We cannot use it to derive the covariances of non-coincident increments of the two processes, since there is no counterpart to identity (2.7). However, for the case of non-overlapping increments the following can be shown directly.

Proposition 3.2 For $0 \leq r_{1}<r_{2} \leq r_{3}<r_{4} \leq 1$,

(i) If $d_{1}+d_{2}>0$,

$$
\begin{aligned}
E\left[\left(X_{2}\left(r_{4}\right)\right.\right. & \left.\left.-X_{2}\left(r_{3}\right)\right)\left(X_{1}\left(r_{2}\right)-X_{1}\left(r_{1}\right)\right)\right] \\
& =\frac{\sigma_{12} U\left(d_{1}, d_{2}\right)}{\Gamma\left(d_{1}+d_{2}+2\right) \sin \pi\left(d_{1}+d_{2}\right)} \sum_{j=1}^{4} \theta_{j} \sin \pi\left(\frac{d_{1}+d_{2}}{2}-\xi_{j}\right)
\end{aligned}
$$

where

$$
U\left(d_{1}, d_{2}\right)=\left(r_{4}-r_{1}\right)^{d_{1}+d_{2}+1}-\left(r_{4}-r_{2}\right)^{d_{1}+d_{2}+1}-\left(r_{3}-r_{1}\right)^{d_{1}+d_{2}+1}+\left(r_{3}-r_{2}\right)^{d_{1}+d_{2}+1}
$$

(ii) If $d_{1}=d_{2}=0$,

$$
E\left[\left(X_{2}\left(r_{4}\right)-X_{2}\left(r_{3}\right)\right)\left(X_{1}\left(r_{2}\right)-X_{1}\left(r_{1}\right)\right)\right]=0 .
$$

To deal with the case $r_{2}>r_{3}$, such that the increments are overlapping, this result may be used in conjunction with (3.3) and the identity

$$
\begin{aligned}
\left(X_{2}\left(r_{4}\right)-X_{2}\left(r_{3}\right)\right)\left(X_{1}\left(r_{2}\right)-X_{1}\left(r_{1}\right)\right)=( & \left.X_{2}\left(r_{4}\right)-X_{2}\left(r_{2}\right)\right)\left(X_{1}\left(r_{2}\right)-X_{1}\left(r_{1}\right)\right) \\
+ & \left.X_{2}\left(r_{2}\right)-X_{2}\left(r_{3}\right)\right)\left(X_{1}\left(r_{2}\right)-X_{1}\left(r_{3}\right)\right) \\
& +\left(X_{2}\left(r_{2}\right)-X_{2}\left(r_{3}\right)\right)\left(X_{1}\left(r_{3}\right)-X_{1}\left(r_{1}\right)\right) .
\end{aligned}
$$

Consider, for example, the case of non-overlapping segments where $X_{1}$ is a backward-looking process, with $\kappa_{1}=1$ and $a_{1}=0$, and $X_{2}$ a forward-looking process, with $\kappa_{2}=0$ and $a_{2}=0$. In this case $\theta_{1}=\theta_{2}=\theta_{4}=0$, and $\xi_{3}=\frac{1}{2}\left(d_{1}+d_{2}\right)$, hence (3.4) vanishes, as we should expect since the lags and leads do not overlap anywhere. However, if $\kappa_{2}=1$ and $\kappa_{1}=0$, so that the segment of the forward-looking process on $r_{2}-r_{1}$ precedes the segment of the backward-looking process on $r_{4}-r_{3}$, then $\theta_{1}=\theta_{2}=\theta_{3}=0$, and the expression in (3.6) reduces to

$$
E\left[\left(X_{2}\left(r_{4}\right)-X_{2}\left(r_{3}\right)\right)\left(X_{1}\left(r_{2}\right)-X_{1}\left(r_{1}\right)\right)\right]=\frac{\sigma_{12} U\left(d_{1}, d_{2}\right)}{\Gamma\left(2+d_{1}+d_{2}\right)}
$$


Observe the symmetry of this formula, being invariant to the interchange of $d_{1}$ and $d_{2}$. In particular, cases where long memory is a property only of the forward-looking process $\left(d_{1}=0\right)$ or of the backward-looking process $\left(d_{2}=0\right)$ are observationally equivalent. However, if both segments are backward looking, such that $\theta_{1}=1$ and $\theta_{2}=\theta_{3}=\theta_{4}=0$, we get

$$
E\left[\left(X_{2}\left(r_{4}\right)-X_{2}\left(r_{3}\right)\right)\left(X_{1}\left(r_{2}\right)-X_{1}\left(r_{1}\right)\right)\right]=\frac{\sigma_{12} U\left(d_{1}, d_{2}\right) \sin \pi d_{2}}{\Gamma\left(2+d_{1}+d_{2}\right) \sin \pi\left(d_{1}+d_{2}\right)} .
$$

which vanishes only if $d_{2}=0$.

\section{Stochastic Integrals with respect to fBM}

While the results of the previous section may be of general interest, it is the distributions of stochastic integrals, random variables of the form $\int_{0}^{1} X_{1} d X_{2}$, that are the chief practical concern in econometric analysis. It is well known that these are not Itô integrals in the case of fractional processes, and their derivation requires methods different from those used in the case of semimartingales such as regular Brownian motion. The problem is addressed in Chan and Terrin (1995) for the particular case they consider, which corresponds effectively ${ }^{3}$ to our case $a_{i}=d_{i} / 2$ and hence arbitrary $\kappa_{i}$. In this section we consider the appropriate generalization of their results.

We follow these authors in defining the differential of $(2.1)$ by replacement of the term $\left(e^{i \lambda r}-\right.$ 1) $/ i \lambda$ with its derivative, so that

$$
\begin{aligned}
\int_{0}^{1} X_{1} d X_{2}=\frac{1}{2 \pi} \int_{0}^{1} d r\left[\int_{-\infty}^{\infty} \frac{e^{i \lambda r}-1}{i \lambda} \tilde{g}\left(\lambda ; d_{1}, \kappa_{1}, a_{1}\right) W_{1}(d \lambda)\right. \\
\\
\left.\times \int_{-\infty}^{\infty} e^{i \mu r} \tilde{g}\left(\mu ; d_{2}, \kappa_{2}, a_{2}\right) W_{2}(d \mu)\right] .
\end{aligned}
$$

For the case $d_{1}+d_{2}>0$, the expected value of this random variable is

$$
E \int_{0}^{1} X_{1} d X_{2}=\frac{\sigma_{12}}{2 \pi} \int_{0}^{1} \int_{-\infty}^{\infty} \frac{1-e^{-i \lambda r}}{i \lambda}|\lambda|^{-d_{1}-d_{2}} P_{12}(\lambda) d \lambda d r
$$

where the form of the last expression can be obtained from (3.2). This formula can be further shown to have the following closed form.

Proposition 4.1 If $d_{1}+d_{2}>0$,

$$
E \int_{0}^{1} X_{1} d X_{2}=\frac{\sigma_{12}}{1+d_{1}+d_{2}} \frac{\Gamma\left(1-d_{1}-d_{2}\right)}{d_{1}+d_{2}} Q_{12}
$$

where

$$
\begin{aligned}
Q_{12}=\frac{1}{\pi}\left[\kappa_{1} \kappa_{2} \sin \pi\left(d_{2}+a_{1}-a_{2}\right)+\left(1-\kappa_{1}\right)\left(1-\kappa_{2}\right) \sin \pi\left(d_{1}+a_{2}-a_{1}\right)\right. \\
\left.\quad+\kappa_{1}\left(1-\kappa_{2}\right) \sin \pi\left(a_{1}+a_{2}\right)+\left(1-\kappa_{1}\right) \kappa_{2} \sin \pi\left(d_{1}+d_{2}-a_{1}-a_{2}\right)\right] .
\end{aligned}
$$

Note the following corollary of Propositions 4.1 and 3.1.

\footnotetext{
${ }^{3}$ Their model allows for slowly varying components which are, however, absorbed into their normalizing sequence, so that the asymptotic analysis is equivalent in the two cases.
} 
Corollary 4.1 If $d_{1}+d_{2}>0$,

$$
E\left(\int_{0}^{1} X_{1} d X_{2}+\int_{0}^{1} X_{2} d X_{1}\right)=E\left(X_{1}(1) X_{2}(1)\right) .
$$

Also note that the case $d_{1}=d_{2}=0$ has to be excluded from these results because our formula in (4.2) then reduces to

$$
E \int_{0}^{1} X_{1} d X_{2}=\frac{\sigma_{12}}{2}+\frac{\sigma_{12}}{2 \pi} \int_{0}^{1} \int_{-\infty}^{\infty} \frac{\sin ^{2} \lambda r}{i \lambda} d \lambda d r .
$$

Although its integrand is an odd function, the integral on the right-hand side has the form $\infty-\infty$, so that the expectation is undefined.

Now consider some contrasting cases of (4.2). We obtain the last term of Chan and Terrin's formula (3.11), apart from scale factor, by setting $a_{j}=d_{j} / 2$ with arbitrary $\kappa_{j}$, for $j=1,2$. In this case we obtain the closed form

$$
E \int_{0}^{1} X_{1} d X_{2}=\frac{\sigma_{12} \Gamma\left(1-d_{1}-d_{2}\right)}{\pi\left(1+d_{1}+d_{2}\right)\left(d_{1}+d_{2}\right)} \sin \pi\left(\frac{d_{2}+d_{1}}{2}\right) .
$$

A feature of this model, consequent on the forward-backward symmetry of the lag structure, is that the formula is symmetric in $d_{1}$ and $d_{2}$. We are not able to distinguish between the case of longer-memory integrand with shorter-memory integrator and the converse case.

In the case of the causal model, in which $\kappa_{1}=\kappa_{2}=1$ and $a_{1}=a_{2}=0$, on the other hand, we obtain

$$
E \int_{0}^{1} X_{1} d X_{2}=\frac{\sigma_{12} \Gamma\left(1-d_{1}-d_{2}\right)}{\pi\left(1+d_{1}+d_{2}\right)\left(d_{1}+d_{2}\right)} \sin \pi d_{2} .
$$

Note, in a result that parallels (3.8), how this quantity vanishes when $d_{2}=0$, although not when $d_{1}=0$.

We next proceed to generalize the second part of Chan and Terrin's Theorem 3.3, which establishes weak convergence of the stochastic integral. Letting $x_{1 t}, x_{2 t}$ be defined like (2.11) with parameters $\left(d_{j}, \kappa_{j}, a_{j}\right)$ for $j=1,2$, our result is as follows.

Theorem 4.1 If $0 \leq d_{1}<\frac{1}{2}, 0 \leq d_{2}<\frac{1}{2}$ and $d_{1}+d_{2}>0$ then

$$
\left(X_{1 n}, X_{2 n,} n^{-1-d_{1}-d_{2}} \sum_{t=1}^{n-1} \sum_{s=1}^{t} x_{1 s} x_{2, t+1}\right) \stackrel{d}{\rightarrow}\left(X_{1}, X_{2}, \int_{0}^{1} X_{1} d X_{2}\right) .
$$

Observe that our conditions here are weaker than the $\min \left(d_{1}, d_{2}\right)>0$ imposed by Chan and Terrin, permitting cases where either of $X_{1}$ and $X_{2}$ are standard Brownian motions. However, the case $d_{1}=d_{2}=0$ is again problematic, not withstanding that the conditions for mean-squared convergence established in the theorem are still satisfied. This follows from the fact noted in (4.5), that the limit specified by formula (4.1) is not an integrable random variable.

The problem arising here can be seen to stem from the approximation of the extreme highfrequency components implicit in the Fourier transformation. This approximation is negligible in the long memory case thanks to non-summability of the autocovariances. In standard Brownian motions, however, it is well known that the equality in (4.4) requires an additional term $\sigma_{12}$ on the left-hand side, representing the contemporaneous correlation of the processes. It is also well known that replacing the partial sum $\sum_{s=1}^{t} x_{1 s}=S_{1 t}$ in (4.6) by $\frac{1}{2}\left(S_{1 t}+S_{1, t+1}\right)$ yields 
the Stratonovich integral ${ }^{4}$ having expectation $\frac{1}{2} \sigma_{12}$, under mean-square convergence in the time domain, instead of the Itô integral having expectation 0. By contrast, an easy implication of our proof is that the limit defined by (4.1) is invariant to finite lead-lag shifts of the integrand sequence in (4.6).

\section{Variants of Fractional Brownian Motion in the Time Domain}

Let $B$ denote Brownian motion on $\mathbb{R}$ with variance $E[B(1)-B(0)]^{2}=\sigma^{2}$. The Gaussian stochastic process on the unit interval $[0,1]$ with representation

$$
X(r)=\frac{1}{\Gamma(d+1)}\left(\int_{0}^{r}(r-s)^{d} d B(s)+\int_{-\infty}^{0}\left[(r-s)^{d}-(-s)^{d}\right] d B(s)\right)
$$

was termed fractional Brownian motion (fBM) by Mandelbrot and van Ness (1968). Robinson and Marinucci (1999) have subsequently dubbed this process fractional Brownian motion of Type I, in contrast with the Type II case in which the second term is discarded. Type II fBM is sometimes rationalized as the weak limit of a process in which the forcing sequence takes the truncated form $u_{t} I(t \geq 1)$. However, it's important to note that the resulting weak limit has a different distribution, and is not to be regarded as an approximation to (5.1).

It is well known (see for example Davidson and de Jong 2000) that the increment variance of Type I fBM is defined by

$$
E[X(r+\delta)-X(r)]^{2}=V \delta^{2 d+1}
$$

where

$$
\begin{aligned}
V=E\left(X(1)^{2}\right) & =\frac{\sigma^{2}}{\Gamma(d+1)^{2}}\left(\frac{1}{2 d+1}+\int_{0}^{\infty}\left[(\tau+1)^{d}-\tau^{d}\right]^{2} d \tau\right) \\
& =\frac{\sigma^{2} \Gamma(1-2 d)}{(2 d+1) \Gamma(1-d) \Gamma(1+d)} .
\end{aligned}
$$

The second equality in (5.2) is a consequence of the following lemma, whose proof we give, for the record, in the Appendix.

Lemma $5.1 \int_{0}^{\infty}\left[(\tau+1)^{d}-\tau^{d}\right]^{2} d \tau=\frac{1}{2 d+1}\left(\frac{\Gamma(1-2 d) \Gamma(1+d)}{\Gamma(1-d)}-1\right)$.

In this section, we extend this time domain representation to a class of the processes whose harmonizable representation is discussed in Section 2. To be precise, we consider just the cases with $0 \leq \kappa \leq 1$ and $a=0$, since for these the extension of (5.1) is simple and transparent. Let

$$
\begin{gathered}
X(r)=\int_{0}^{r}\left[\kappa(r-s)^{d}+(1-\kappa) s^{d}\right] d B(s)+\kappa \int_{-\infty}^{0}\left[(r-s)^{d}-(-s)^{d}\right] d B(s) \\
+(1-\kappa) \int_{r}^{\infty}\left[s^{d}-(s-r)^{d}\right] d B(s), \quad 0 \leq r \leq 1 .
\end{gathered}
$$

Defining the filtration

$$
\mathcal{F}=\{\mathcal{F}(r)=\sigma(B(s), s \leq r),-\infty<r<\infty\}
$$

note that $(X, \mathcal{F})$ are an adapted pair in the case $\kappa=1$, which corresponds to (5.1). This is another way to express the notion of a causal process. Otherwise, $X$ is $\mathcal{F}(\infty)$-measurable at all

\footnotetext{
${ }^{4}$ See, inter alia, Duncan, Hu and Pasik Duncan (2000).
} 
points, and hence not adapted to $\mathcal{F}$. With $\kappa=\frac{1}{2}$, such that the forward and backward looking moving averages are symmetric, note that this model corresponds to case (2.10), while with $\kappa=1$ it corresponds to (2.8).

We remark parenthetically that there appears no natural way to define a 'Type II' variant of this class of model. Thus, the process $\{X(r), 0 \leq r \leq 1\}$ obtained by deleting the second and third terms of (5.3) corresponds to the weak limit of a fractionally integrated process whose forcing sequence takes the form $u_{t} I(1 \leq t \leq[n r])$ for each $r$. One could truncate the forcing sequence at $n$ and hence the third integral at 1, instead of deleting it, but this option foregoes the benefits of simplicity and seems equally arbitrary. There are many ways to construct stochastic processes having correlated increments, subject to a parameter $d$, but not all of them have relevance to econometric modelling.

The next proposition derives the general formula for the increment variances.

Proposition 5.1 $E[X(r+\delta)-X(r)]^{2}=V \delta^{2 d+1}$ where

$$
V=\frac{\sigma^{2}}{\Gamma(1+d)(2 d+1)}\left(2 \kappa(1-\kappa) \frac{\Gamma(1+d)}{\Gamma(1+2 d)}+(1-2 \kappa(1-\kappa)) \frac{\Gamma(1-2 d)}{\Gamma(1-d)}\right) .
$$

We need to check the relationship between this formula and (2.6) obtained in the harmonizable representation for the case $a=0$. The following proposition shows they are identical.

Proposition 5.2 $V=\frac{\sigma^{2} K(d, \kappa, 0)^{2}}{\Gamma(2 d+2) \cos \pi d}$.

The main implication to be remarked here is that the Mandelbrot-Van Ness model (5.1) is shown to be equivalent to the causal variant (2.8) of the harmonizable representation, these being Gaussian processes with identical covariance structure. This fact may suggest a further need for caution in adopting the Type II variant of time domain fBM in the causal model. Having nonstationary increments, the Type II variant evidently does not possess a harmonizable representation.

Since empirical processes will always be subject to an unknown scale factor $\sigma$, it is not possible to distinguish the processes represented by (5.3) from one another in isolation, and $\kappa$ is unidentified. However, similar arguments allow us to show that for processes $X_{1}$ and $X_{2}$ the covariances of process segments, and $E \int_{0}^{1} X_{1} d X_{2}$, depend on $\kappa_{1}, \kappa_{2}$. This can be verified simply by considering the harmonizable representations in Sections 3 and 4 , which yield equivalent formulae.

It is known (Davidson and de Jong 2000, Theorem 3.1) that if $x_{t}=(1-L)^{-d} u_{t}$, where $\left\{u_{t}\right\}$ satisfies fairly weak regularity conditions and

$$
X_{n}(r)=n^{-1 / 2-d} \sum_{t=1}^{[n t]} x_{t}
$$

then $X_{n} \stackrel{d}{\rightarrow} X$ where $X$ is defined by (5.1), and $\stackrel{d}{\rightarrow}$ here denotes weak convergence in the space $D_{[0,1]}$ of cadlag functions on the unit interval, equipped with the Skorokhod topology (see, e.g., Davidson 1994, Ch. 28). We can now extend this result as follows.

Theorem 5.1 If

$$
x_{t}=\left[\kappa(1-L)^{-d}+(1-\kappa)\left(1-L^{-1}\right)^{-d}\right] u_{t}
$$

for any $d \in\left(-\frac{1}{2}, \frac{1}{2}\right)$ and $\kappa \in[0,1]$, and $X_{n}$ is defined in (5.4), then $X_{n} \stackrel{d}{\rightarrow} X$ under the assumptions on $u_{t}$ specified by Davidson and de Jong's (2000) Theorem 3.1. 
We refer the reader to the cited source for details of the specified conditions, which are quite complicated to state. It suffices to say that they specify weak dependence in the form of $L_{2}$ near-epoch dependence on a mixing process, at specified sizes. These conditions are invariant to time reversal, which allows the extension of the results specified in the proof of the present proposition. Also note that this proposition is not redundant in view of Theorem 2.1, not merely because it covers the cases $d<0$, but because it allows a very general class of weakly dependent shock processes. While it is possible to introduce additional weak dependence in Theorem 2.1 (we avoid this for the sake of simplicity) note that Gaussianity is a necessary condition, in the spectral approach.

\section{Discussion}

In the literature on fractional Brownian motion, there is a tendency to assume the existence of a single Gaussian process having the characteristics of interest. In the time domain, the formulation most often cited is the one of Mandelbrot and van Ness (1968). Among many references in the engineering, statistics and econometrics literature see Flandrin (1989), Samorodnitsky and Taqqu (1994, Sect. 7.2), Read, Lee and Truong (1995).

However, there is less consensus regarding the so-called harmonizable representation of fractional Brownian motion. Samorodnitsky and Taqqu (1994, Sect. 7.2), which is, perhaps, the chief reference source on these topics, refers to one of the 'time-symmetric' members of our class, that having $a=d / 2$ and arbitrary $\kappa$, as the (integral representation of) 'standard' fractional Brownian motion, and Chan and Terrin (1995) follow suit. On the other hand, Reed et al. (1995) cite the 'causal' form $(\kappa=1, a=0)$. Kim and Phillips (2001) actually cite the causal version in the statement of their model, but then switch to the time symmetric case in their working, while following the Chan-Terrin analysis. This apparent confusion is very likely attendant on the fact that the underlying stationary driving processes all have the same spectrum. Indeed, Chan and Terrin (1995) explicitly set up their model by citing the spectral density of interest $\left(|\lambda|^{1-2 H}\right.$ multiplied by a possibly slowly varying scale component), and then selecting the square root of this function as the transfer function to define their harmonizable representation.

However, as we have shown in this paper, the Mandelbrot-Van Ness time domain model and the time-symmetric model discussed by Samorodnitsky-Taqqu, Chan-Terrin and other authors, have very different implications for multivariate analysis, despite the stationary increment processes sharing the same spectrum, apart from scale constants. We note that Chan-Terrin invoke econometric applications explicitly in their introduction, introduce a unit root autoregressive (backward-looking) model, and even quote the one-sided time domain representation $(1-L)^{d} y_{t}$ to motivate their formal analysis of the long memory errors. This suggests that they did not fully appreciate the modelling implications of their chosen harmonizable representation. When the applications of interest are in time series econometrics, we ought to point out that for most applications only a single member of our class should generally be considered; the causal (i.e. exclusively backward-looking) Mandelbrot-Van Ness model, with harmonizable representation (2.8) having $\kappa=1, a=0$ in the Gaussian case. Time-series processes in econometrics are almost always thought of as adapted to the filtration representing 'events to present date', since any other setup would contradict our usual understanding of economic behaviour. ${ }^{5}$

Frequency-domain analysis is a popular research methodology in time series econometrics, especially in the analysis of long memory and fractional integration and cointegration. We can cite for example the many contributions of Peter Robinson and co-authors; Robinson (1994a,b),

\footnotetext{
${ }^{5}$ Two-sided models could have applications in random field representations involving spatial correlations, for example.
} 
Robinson and Marinucci (2001, 2003), Marinucci and Robinson (1999, 2000, 2001) is a nonexhaustive list covering just one aspect of this research. One obvious motivation for this methodology is the greater mathematical tractability often available in the asymptotic analysis of long memory models. By the same token, however, we see a need to emphasize the potential pitfalls of modelling dynamic relationships in a framework that may conceal the important role of causal orderings.

\section{A Appendix: Proofs}

\section{A.1 Proof of Theorem 2.1}

Our proof is based on the first part of Chan and Terrin's (1995) Theorem 3.3 (henceforth, CT). We modify their notation by substituting $d+\frac{1}{2}$ for $H_{1}, n^{-1 / 2-d}$ for $a_{n}, \sqrt{2 \pi} X_{n}$ for $a_{n}^{-1} U_{n}$, and $\sqrt{2 \pi} \sigma^{-1} K(d, \kappa, a)^{-1} X$ for $K_{H_{1}}^{-1} B_{H_{1}}$ where they define ${ }^{6}$

$$
K_{H_{1}}^{-1} B_{H_{1}}(t)=\int_{-\infty}^{\infty} \frac{e^{i \lambda t}-1}{i \lambda}|\lambda|^{1 / 2-H_{1}} W(d \lambda)
$$

and $K_{H_{1}}=\left[\pi^{-1} H_{1} \Gamma\left(2 H_{1}\right) \sin \pi H_{1}\right]^{1 / 2}$. We neglect the possibility of slowly varying components, which in practice must be incorporated in the normalizing sequence $a_{n}$, thereby obtaining the same limiting formulae for moments, etc..

Following CT, we cite Taqqu (1975) Theorem 2.1 to establish tightness of the sequence, and accordingly prove weak convergence of the finite dimensional distributions by showing that

$$
E\left|X_{n}(r)-X(r)\right|^{2} \rightarrow 0
$$

for each $r \in[0,1]$. Note that CT's expression for $U_{n}$ (p. 1672, line 7 from bottom) is generalized to our class by replacing $|\lambda|^{1 / 2-H_{1}}$ by $\tilde{g}(\lambda ; d, \kappa, a)$, which entails inserting the factor $\Upsilon(\lambda ; d, \kappa, a)$ defined by (2.4). CT's case is $\tilde{g}(\lambda ; d, 1, d / 2)$ where $\Upsilon(\lambda ; d, \kappa, a)=1$. However, their expressions

$$
D_{n}(r, \lambda)=1_{[-n \pi, n \pi]}(\lambda) \frac{\exp [i \lambda([n r]+1) / n])-\exp (i \lambda / n)}{n(\exp (i \lambda / n)-1)}
$$

and

$$
D(r, \lambda)=\frac{\exp (i r \lambda)-1}{i \lambda}
$$

carry over unchanged. Therefore, we need to demonstrate that the following sequence (the counterpart of CT equation (3.12)), must converge to 0 as $n \rightarrow \infty$.

$$
\begin{aligned}
\frac{2 \pi}{\sigma^{2}} E\left|X_{n}(r)-X(r)\right|^{2} & =\int_{-\infty}^{\infty}\left|D_{n}(r, \lambda) n^{-d} g\left(e^{-i \lambda / n} ; d, \kappa, a\right)-D(r, \lambda) \tilde{g}(\lambda ; d, \kappa, a)\right|^{2} d \lambda \\
\leq K(d, \kappa, a)^{2} & \int_{-\infty}^{\infty}\left|D_{n}(r, \lambda)-D(r, \lambda)\right|^{2}|\lambda|^{-2 d} d \lambda \\
& \quad+\int_{-\infty}^{\infty}\left|D_{n}(r, \lambda)\right|^{2}\left|n^{-d} g\left(e^{-i \lambda / n}\right)-\tilde{g}(\lambda)\right|^{2} d \lambda
\end{aligned}
$$

\footnotetext{
${ }^{6}$ The definition of the normalizing constant $K_{H 1}$ will depend on the member of the class of processes we consider, of which CT's case is only one, but we will not state these explicitly. Be careful to note that CT assume $W$ has unit variance, and also note the error in their definition of $K_{H_{1}}$, omitting the square root (line following (3.10) on CT page 1671).
} 
Since $D_{n}(r, \lambda) \rightarrow D(r, \lambda)$ pointwise on $[0,1] \times \mathbb{R}$, to show the first majorant term vanishes it suffices to show that

$$
\int_{-\infty}^{\infty}\left|D_{n}(r, \lambda)\right|^{2}|\lambda|^{-2 d} d \lambda=\int_{-\pi n}^{\pi n}\left|D_{n}(r, \lambda)\right|^{2}|\lambda|^{-2 d} d \lambda<\infty .
$$

uniformly in $n$. Note first that

$$
\begin{aligned}
\left|D_{n}(r, \lambda)\right|^{2} & =1_{[-n \pi, n \pi]}\left|\frac{\exp [i \lambda([n r]+1) / n]-\exp (i \lambda / n)}{n(\exp (i \lambda / n)-1)}\right|^{2} \\
& =1_{[-n \pi, n \pi]} \frac{|\exp [i \lambda([n r]+1) / n]-\exp (i \lambda / n)|^{2}}{|n(\exp (i \lambda / n)-1)|^{2}} \\
& =1_{[-n \pi, n \pi]} \frac{1}{n^{2}} \frac{(\exp [i \lambda([n r]+1) / n]-\exp (i \lambda / n))(\exp [i \lambda([n r]+1) / n]-\exp (i \lambda / n))}{(\exp (i \lambda / n)-1)(\exp (-i \lambda / n)-1)} \\
& =1_{[-n \pi, n \pi]} \frac{1}{n^{2}} \frac{2-2 \cos \lambda[n r] / n}{2-2 \cos \lambda / n} \\
& =1_{[-n \pi, n \pi]} \frac{1}{n^{2}} \frac{\sin ^{2} \lambda[n r] / 2 n}{\sin ^{2} \lambda / 2 n} .
\end{aligned}
$$

Therefore, using the results

$$
\frac{\sin ^{2} k x}{\sin ^{2} x} \leq k^{2}
$$

and

$$
\int_{0}^{\pi / 2} \frac{\sin ^{2} k x}{\sin ^{2} x} d x=\frac{\pi k}{2}
$$

for integer $k \geq 0$, observe that

$$
\begin{aligned}
\int_{-\pi n}^{\pi n}\left|D_{n}(r, \lambda)\right|^{2}|\lambda|^{-2 d} d \lambda & =\int_{-\pi n}^{\pi n} \frac{1}{n^{2}} \frac{\sin ^{2} \lambda[n r] / 2 n}{\sin ^{2} \lambda / 2 n}|\lambda|^{-2 d} d \lambda \\
& =2 \int_{0}^{\pi n} \frac{1}{n^{2}} \frac{\sin ^{2} \lambda[n r] / 2 n}{\sin ^{2} \lambda / 2 n} \lambda^{-2 d} d \lambda \\
& =2\left(\int_{0}^{1} \frac{1}{n^{2}} \frac{\sin ^{2} \lambda[n r] / 2 n}{\sin ^{2} \lambda / 2 n} \lambda^{-2 d} d \lambda+\int_{1}^{\pi n} \frac{1}{n^{2}} \frac{\sin ^{2} \lambda[n r] / 2 n}{\sin ^{2} \lambda / 2 n} \lambda^{-2 d} d \lambda\right) \\
& \leq 2\left(\int_{0}^{1} \frac{[n r]^{2}}{n^{2}} \lambda^{-2 d} d \lambda+\int_{1}^{\pi n} \frac{1}{n^{2}} \frac{\sin ^{2} \lambda[n r] / 2 n}{\sin ^{2} \lambda / 2 n} \lambda^{-2 d} d \lambda\right) \\
& \leq 2\left(\frac{1}{1-2 d}+\int_{1}^{\pi n} \frac{1}{n^{2}} \frac{\sin ^{2} \lambda[n r] / 2 n}{\sin ^{2} \lambda / 2 n} \lambda^{-2 d} d \lambda\right) \\
& \leq 2\left(\frac{1}{1-2 d}+\int_{0}^{\pi n} \frac{\sin ^{2} \lambda[n r] / 2 n}{\sin ^{2} \lambda / 2 n} d \lambda\right) \\
& =2\left(\frac{1}{1-2 d}+\frac{1}{n^{2}} 2 n \frac{\pi}{2}[n r]\right) \\
& \leq 2\left(\frac{1}{1-2 d}+\pi\right) .
\end{aligned}
$$

To show that the second majorant term in (A-1) vanishes as $n \rightarrow \infty$, note that

$$
\left|n^{-d} g\left(e^{-i \lambda / n}\right)-\tilde{g}(\lambda)\right|^{2}
$$




$$
\begin{aligned}
=\mid \kappa[(i \lambda & \left.\left.+O\left(n^{-1}\right)\right)^{a-d}\left(-i \lambda+O\left(n^{-1}\right)\right)^{-a}-(i \lambda)^{a-d}(-i \lambda)^{-a}\right] \\
& +\left.(1-\kappa)\left[\left(i \lambda+O\left(n^{-1}\right)\right)^{-a}\left(-i \lambda+O\left(n^{-1}\right)\right)^{a-d}-(i \lambda)^{-a}(-i \lambda)^{a-d}\right]\right|^{2} .
\end{aligned}
$$

Taking the first right-hand side term under the modulus in (A-5), note that

$$
\left(i \lambda+O\left(n^{-1}\right)\right)^{a-d}\left(-i \lambda+O\left(n^{-1}\right)\right)^{-a}-(i \lambda)^{a-d}(-i \lambda)^{-a}=|\lambda|^{-d} \varpi_{1 n}(\lambda, a, d) .
$$

where

$$
\begin{aligned}
\varpi_{1 n}(\lambda, a, d) & =\left[\left(e^{i \pi \operatorname{sgn}(\lambda) / 2}+O\left(n^{-1}\right)\right)^{a-d}\left(e^{-i \pi \operatorname{sgn}(\lambda) / 2}+O\left(n^{-1}\right)\right)^{-a}-e^{-i \pi(d / 2-a) \operatorname{sgn}(\lambda)}\right] \\
& =O\left(n^{-1}\right) .
\end{aligned}
$$

The same treatment of the second right-hand side term yields an expression $\varpi_{2 n}(\lambda, a, d)=O\left(n^{-1}\right)$ similarly. Note that $\varpi_{1 n}(\lambda, a, d)$ and $\varpi_{2 n}(\lambda, a, d)$ depend on $\lambda$ only through its sign, and hence the squared modulus in (A-5) depends on $\lambda$ only through $|\lambda|^{-2 d}$. We therefore complete the proof by applying (A-4) as before.

\section{A.2 Proof of Theorem 2.2}

We show that $E\left[X_{n}(r)^{2}\right] \rightarrow E\left[X(r)^{2}\right]$ for each $r \in[0,1]$. As remarked in the text, this condition is sufficient for the weak convergence, since the sequence members are linear and Gaussian for each $n \geq 1$ and uniform tightness holds as before, by appeal to Taqqu (1975 Th. 2.1).

The spectral representation of $X_{n}(r)$ is

$$
\begin{aligned}
X_{n}(r) & =\frac{1}{n^{1 / 2+d}} \sum_{t=1}^{[n r]} \frac{1}{\sqrt{2 \pi}} \int_{-\pi}^{\pi} e^{i \lambda t}\left(1-e^{-i \lambda}\right)^{-d} W(d \lambda) \\
& =\frac{1}{n^{1 / 2+d} \sqrt{2 \pi}} \int_{-\pi}^{\pi} \sum_{t=1}^{[n r]} e^{i \lambda t}\left(1-e^{-i \lambda}\right)^{-d} W(d \lambda) \\
& =\frac{1}{n^{1 / 2+d} \sqrt{2 \pi}} \int_{-\pi}^{\pi} e^{i \lambda} \frac{e^{i \lambda[n r]}-1}{e^{i \lambda}-1}\left(1-e^{-i \lambda}\right)^{-d} W(d \lambda) \\
& =\frac{1}{n^{1 / 2+d} \sqrt{2 \pi}} \int_{-\pi}^{\pi}\left(e^{i \lambda[n r]}-1\right)\left(1-e^{-i \lambda}\right)^{-d-1} W(d \lambda) .
\end{aligned}
$$

The integrand can be rewritten as

$$
\begin{aligned}
\left(e^{i \lambda[n r]}-1\right) & \left(1-e^{-i \lambda}\right)^{-d-1} \\
& =e^{i \lambda[n r] / 2} 2 i \sin \frac{\lambda[n r]}{2}\left(e^{-i \lambda / 2} 2 i \sin \frac{\lambda}{2}\right)^{-d-1} \\
& =2^{-d} e^{i \lambda([n r]+d+1) / 2}(i \operatorname{sgn} \lambda)^{-d} \sin \frac{|\lambda|[n r]}{2} \sin ^{-d-1} \frac{|\lambda|}{2} \\
& =2^{-d} e^{i \lambda([n r]+d+1) / 2} e^{-i \operatorname{sgn} \lambda \pi d / 2} \sin \frac{|\lambda|[n r]}{2} \sin ^{-d-1} \frac{|\lambda|}{2}
\end{aligned}
$$

The variance of $X_{n}(r)$ is therefore equal to

$$
V_{n}(r)=\frac{\sigma^{2}}{2 \pi} \frac{2^{1-2 d}}{n^{1+2 d}} \int_{0}^{\pi} \sin ^{2} \frac{\lambda[n r]}{2} \sin ^{-2 d-2} \frac{\lambda}{2} d \lambda
$$




$$
\begin{aligned}
& =\frac{\sigma^{2}}{2 \pi} \frac{2^{1-2 d}}{n^{1+2 d}} \int_{0}^{\pi / 2} 2 \sin ^{2} \varphi[n r] \sin ^{-2 d-2} \varphi d \varphi \\
& =\frac{\sigma^{2}}{2 \pi} \frac{2^{1-2 d}}{n^{1+2 d}} \int_{0}^{\pi / 2}(1-\cos 2 \varphi[n r]) \sin ^{-2 d-2} \varphi d \varphi
\end{aligned}
$$

By GR Relation 3.621(1),

$$
\begin{aligned}
\int_{0}^{\pi / 2} \sin ^{-2 d-2} \varphi d \varphi & =2^{-2 d-3} B\left(-d-\frac{1}{2},-d-\frac{1}{2}\right) \\
& =\frac{\pi d}{2 d+1} \frac{\Gamma(1-2 d)}{[\Gamma(1-d)]^{2}}
\end{aligned}
$$

where the second equality follows after some manipulation using the properties of the gamma function. Next,

$$
\begin{aligned}
\int_{0}^{\pi / 2} \cos 2 \varphi[n r] \sin ^{-2 d-2} \varphi d \varphi & =\int_{0}^{\pi / 2} \cos 2\left(\frac{\pi}{2}-\varphi\right)[n r] \cos ^{-2 d-2} \varphi d \varphi \\
& =\int_{0}^{\pi / 2} \cos (\pi[n r]-2[n r] \varphi) \cos ^{-2 d-2} \varphi d \varphi \\
& =(-1)^{[n r]} \int_{0}^{\pi / 2} \cos (2[n r] \varphi) \cos ^{-2 d-2} \varphi d \varphi \\
& =(-1)^{[n r]} \frac{\pi}{2^{-2 d-1}(-2 d-1)} \frac{1}{B(-d+[n r],-d-[n r])} \\
& =-\frac{2^{2 d}}{2 d+1} \frac{\pi}{\cos \pi d} \frac{\Gamma(1+d+[n r])}{\Gamma(1+2 d) \Gamma(-d+[n r])}
\end{aligned}
$$

where the penultimate equality follows from GR Relation 3.631(9). Upon substitution of these expressions into the expression for the variance we obtain

$$
V_{n}(r)=\frac{\sigma^{2}}{2 \pi} \frac{2^{1-2 d}}{n^{1+2 d}}\left(\frac{\pi d \Gamma(1-2 d)}{(2 d+1)[\Gamma(1-d)]^{2}}+\frac{2^{2 d}}{2 d+1} \frac{\pi}{\cos \pi d} \frac{\Gamma(1+d+[n r])}{\Gamma(1+2 d) \Gamma(-d+[n r])}\right) .
$$

In the limit, as $n \rightarrow \infty$, the contribution of the first term in the brackets vanishes. For the second term we use

$$
\lim _{n \rightarrow \infty} \frac{\Gamma(1+d+[n r])}{\Gamma(-d+[n r])}[n r]^{-2 d-1}=1
$$

Hence,

$$
\lim _{n \rightarrow \infty} V_{n}(r)=\frac{\sigma^{2}}{\Gamma(2+2 d) \cos \pi d} r^{2 d+1}
$$

The right-hand side is equal to the variance of the fBM process

$$
X(r)=\frac{1}{\sqrt{2 \pi}} \int_{-\infty}^{\infty} \frac{e^{i \lambda r}-1}{i \lambda}(i \lambda)^{-d} W(d \lambda) .
$$

To show this observe that the integrand can be rewritten as

$$
\begin{aligned}
\frac{e^{i \lambda r}-1}{i \lambda}(i \lambda)^{-d} & =e^{i \lambda r / 2} 2 i \sin \frac{\lambda r}{2}(i \lambda)^{-d-1} \\
& =2 e^{i \lambda r / 2} \sin \frac{|\lambda| r}{2}|\lambda|^{-d-1}(i \operatorname{sgn} \lambda)^{-d}
\end{aligned}
$$




$$
=2 e^{i \lambda r / 2} e^{-i \operatorname{sgn} \lambda \pi d / 2} \sin \frac{|\lambda|}{2}|\lambda|^{-d},
$$

and, hence, the variance of $X(r)$ takes the form

$$
\begin{aligned}
V(r) & =\frac{2 \sigma^{2}}{\pi} \int_{-\infty}^{\infty} \sin ^{2} \frac{|\lambda| r}{2}|\lambda|^{-2 d-2} d \lambda \\
& =\frac{2 \sigma^{2}}{\pi} \int_{0}^{\infty}(1-\cos \lambda r) \lambda^{-2 d-2} d \lambda \\
& =\frac{\sigma^{2}}{\Gamma(2+2 d) \cos \pi d} r^{2 d+1}
\end{aligned}
$$

where the last equality follows from GR Relation 3.823.

\section{A.3 Proof of Proposition 3.1}

$$
\begin{aligned}
E\left[\left(X_{2}(r+\delta)\right.\right. & \left.\left.-X_{2}(r)\right)\left(X_{1}(r+\delta)-X_{1}(r)\right)\right] \\
& =\frac{\sigma_{12}}{\pi} \int_{-\infty}^{\infty}|\lambda|^{-d_{1}-d_{2}-2}(1-\cos \lambda \delta) P_{12}(\lambda) d \lambda \\
& =\frac{\sigma_{12} \delta^{d_{1}+d_{2}+1}}{\pi} \int_{-\infty}^{\infty}|x|^{-d_{1}-d_{2}-2}(1-\cos x) P_{12}(x) d x
\end{aligned}
$$

Substituting from (3.2), note that the last integral has four terms, with typical form

$$
\begin{aligned}
\int_{-\infty}^{\infty}|x|^{-d_{1}-d_{2}-2}(1-\cos x) e^{i \pi \xi_{j} \operatorname{sgn}(x)} d x & =2 \cos \pi \xi_{j} \int_{0}^{\infty} d x \frac{1-\cos x}{x^{2+d_{1}+d_{2}}} \\
& =2 \cos \pi \xi_{j} \frac{\pi \csc \frac{\pi}{2}\left(d_{1}+d_{2}+1\right)}{2 \Gamma\left(2+d_{1}+d_{2}\right)} \\
& =\frac{\pi}{\Gamma\left(2+d_{1}+d_{2}\right)} \frac{\cos \pi \xi_{j}}{\cos \pi \frac{d_{1}+d_{2}}{2}}
\end{aligned}
$$

(see GR, relations 3.761 and 3.823). Direct substitution into (A-7) gives (3.3).

\section{A.4 Proof of Proposition 3.2}

$$
\begin{aligned}
E\left[\left(X_{2}\left(r_{4}\right)-\right.\right. & \left.\left.X_{2}\left(r_{3}\right)\right)\left(X_{1}\left(r_{2}\right)-X_{1}\left(r_{1}\right)\right)\right] \\
& =\frac{\sigma_{12}}{2 \pi} \int_{-\infty}^{\infty} \frac{e^{i \lambda r_{4}}-e^{i \lambda r_{3}}}{i \lambda} \widetilde{g}\left(\lambda ; d_{2}, \kappa_{2}, a_{2}\right) \frac{e^{-i \lambda r_{2}}-e^{-i \lambda r_{1}}}{-i \lambda} \widetilde{g}\left(-\lambda ; d_{1}, \kappa_{1}, a_{1}\right) d \lambda \\
& =\frac{\sigma_{12}}{2 \pi} \int_{-\infty}^{\infty}|\lambda|^{-d_{1}-d_{2}-2}\left(e^{i \lambda r_{4}}-e^{i \lambda r_{3}}\right)\left(e^{-i \lambda r_{2}}-e^{-i \lambda r_{1}}\right) P_{12}(\lambda) d \lambda .
\end{aligned}
$$

Rearranging the terms with the exponents gives

$$
\begin{aligned}
\left(e^{i \lambda r_{4}}-e^{i \lambda r_{3}}\right) & \left(e^{-i \lambda r_{2}}-e^{-i \lambda r_{1}}\right) \\
& =e^{i \lambda\left(r_{4}+r_{3}\right) / 2} e^{-i \lambda\left(r_{2}+r_{1}\right) / 2}\left(e^{i \lambda\left(r_{4}-r_{3}\right) / 2}-e^{-i \lambda\left(r_{4}-r_{3}\right) / 2}\right)\left(e^{-i \lambda\left(r_{2}-r_{1}\right) / 2}-e^{i \lambda\left(r_{2}-r_{1}\right) / 2}\right) \\
& =4 e^{i \lambda\left[\left(r_{4}+r_{3}\right)-\left(r_{2}+r_{1}\right)\right] / 2} \sin \lambda \frac{r_{4}-r_{3}}{2} \sin \lambda \frac{r_{2}-r_{1}}{2} .
\end{aligned}
$$


Next, define integrals

$$
\begin{aligned}
& \Phi_{1}=\int_{0}^{\infty} \frac{d \lambda}{\lambda^{d_{1}+d_{2}+2}} \cos \lambda \frac{\left(r_{4}+r_{3}\right)-\left(r_{2}+r_{1}\right)}{2} \sin \lambda \frac{r_{4}-r_{3}}{2} \sin \lambda \frac{r_{2}-r_{1}}{2} \\
& \Phi_{2}=\int_{0}^{\infty} \frac{d \lambda}{\lambda^{d_{1}+d_{2}+2}} \sin \lambda \frac{\left(r_{4}+r_{3}\right)-\left(r_{2}+r_{1}\right)}{2} \sin \lambda \frac{r_{4}-r_{3}}{2} \sin \lambda \frac{r_{2}-r_{1}}{2}
\end{aligned}
$$

and note that

$$
\Phi_{1}+i \Phi_{2}=\int_{0}^{\infty} \frac{e^{i \lambda\left[\left(r_{4}+r_{3}\right)-\left(r_{2}+r_{1}\right)\right] / 2}}{\lambda^{d_{1}+d_{2}+2}} \sin \lambda \frac{r_{4}-r_{3}}{2} \sin \lambda \frac{r_{2}-r_{1}}{2} d \lambda .
$$

The expression for the covariance can accordingly be rewritten as

$$
\begin{aligned}
E\left[\left(X_{2}\left(r_{4}\right)-\right.\right. & \left.\left.X_{2}\left(r_{3}\right)\right)\left(X_{1}\left(r_{2}\right)-X_{1}\left(r_{1}\right)\right)\right] \\
& =\frac{2 \sigma_{12}}{\pi} \sum_{j=1}^{4} \theta_{j} \int_{-\infty}^{\infty} \frac{e^{i \pi \xi_{j} \operatorname{sgn}(\lambda)} e^{i \lambda\left[\left(r_{4}+r_{3}\right)-\left(r_{2}+r_{1}\right)\right] / 2}}{|\lambda|^{d_{1}+d_{2}+2}} \sin \lambda \frac{r_{4}-r_{3}}{2} \sin \lambda \frac{r_{2}-r_{1}}{2} d \lambda \\
& =\frac{2 \sigma_{12}}{\pi} \sum_{j=1}^{4} \theta_{j}\left[e^{i \pi \xi_{j}}\left(\Phi_{1}+i \Phi_{2}\right)+e^{-i \pi \xi_{j}}\left(\Phi_{1}-i \Phi_{2}\right)\right] \\
& =\frac{4 \sigma_{12}}{\pi} \sum_{j=1}^{4} \theta_{j}\left(\Phi_{1} \cos \pi \xi_{j}-\Phi_{2} \sin \pi \xi_{j}\right)
\end{aligned}
$$

The integrand in $\Phi_{1}$ can be rearranged using a trigonometric identity as

$$
\begin{aligned}
\cos \lambda & \frac{\left(r_{4}+r_{3}\right)-\left(r_{2}+r_{1}\right)}{2} \sin \lambda \frac{r_{4}-r_{3}}{2} \sin \lambda \frac{r_{2}-r_{1}}{2} \\
& =\frac{1}{2} \sin \lambda\left(r_{4}-\frac{r_{2}+r_{1}}{2}\right) \sin \lambda \frac{r_{2}-r_{1}}{2}-\frac{1}{2} \sin \lambda\left(r_{3}-\frac{r_{2}+r_{1}}{2}\right) \sin \lambda \frac{r_{2}-r_{1}}{2} .
\end{aligned}
$$

Applying GR Relation 3.762(1) then yields

$$
\Phi_{1}=\frac{\pi \sec \pi \frac{d_{1}+d_{2}}{2}}{8 \Gamma\left(d_{1}+d_{2}+2\right)} U\left(d_{1}, d_{2}\right)
$$

where $U\left(d_{1}, d_{2}\right)$ is given by (3.5). Note that $\Phi_{1}=0$ for $d_{1}+d_{2}=0$.

For $\Phi_{2}$, use GR Relations 3.763(1) and (3) to obtain

$$
\Phi_{2}= \begin{cases}\frac{\pi \csc \pi \frac{d_{1}+d_{2}}{2}}{8 \Gamma\left(d_{1}+d_{2}+2\right)} U\left(d_{1}, d_{2}\right), & d_{1}+d_{2}>0, \\ \frac{1}{4}\left[\left(r_{4}-r_{1}\right) \ln \left(r_{4}-r_{1}\right)-\left(r_{4}-r_{2}\right) \ln \left(r_{4}-r_{2}\right)\right. & \\ \left.+\left(r_{3}-r_{1}\right) \ln \left(r_{3}-r_{1}\right)-\left(r_{3}-r_{2}\right) \ln \left(r_{3}-r_{2}\right)\right], & d_{1}+d_{2}=0 .\end{cases}
$$

Substituting these results in (A-9) yields (3.4) for the case $d_{1}+d_{2}>0$. The case $d_{1}=d_{2}=0$ implies $a_{1}=a_{2}=0$. Hence, $\xi_{j}=0$ for $j=1,2,3,4$ and

$$
\begin{aligned}
E\left[\left(X_{2}\left(r_{4}\right)-X_{2}\left(r_{3}\right)\right)\left(X_{1}\left(r_{2}\right)-X_{1}\left(r_{1}\right)\right)\right] & =-\frac{4 \sigma_{12} \Phi_{2}}{\pi} \sum_{j=1}^{4} \theta_{j} \sin \pi \xi_{j} \\
& =0 .
\end{aligned}
$$




\section{A.5 Proof of Proposition 4.1}

Since $0 \leqslant r \leqslant 1$ we have $\operatorname{sgn}(\lambda)=\operatorname{sgn}(\lambda r)$, and making the change of variables $x=\lambda r$ we can integrate with respect to $r$ independently. This yields the general formula

$$
\begin{aligned}
E \int_{0}^{1} X_{1} d X_{2} & =\frac{\sigma_{12}}{2 \pi} \int_{0}^{1} d r r^{d_{1}+d_{2}} \int_{-\infty}^{\infty} \frac{1-e^{-i x}}{i x}|x|^{-d_{1}-d_{2}} P_{12}(x) d x \\
& =\frac{\sigma_{12}}{2 \pi} \frac{1}{1+d_{1}+d_{2}} \int_{-\infty}^{\infty} \frac{1-e^{-i x}}{i x}|x|^{-d_{1}-d_{2}} P_{12}(x) d x
\end{aligned}
$$

where $P_{12}$ is defined in (3.2), containing terms of the type $\exp \left(i \pi \xi_{j} \operatorname{sgn}(\lambda)\right)$, where $\xi_{j}$ is one of the indicated functions of $d_{1}, d_{2}, a_{1}, a_{2}$. For any such term we can rewrite the corresponding component of the integral over the real line in (A-10) (say, $C\left(\xi_{j}\right)$ ), as

$$
\begin{aligned}
C\left(\xi_{j}\right) & \equiv \int_{-\infty}^{\infty} d x \frac{1-e^{-i x}}{i x}|x|^{-d_{1}-d_{2}} \exp \left(i \pi \xi_{j} \operatorname{sgn}(\lambda)\right) \\
& =\int_{0}^{\infty} d x \frac{1-e^{-i x}}{i x}|x|^{-d_{1}-d_{2}} \exp \left(i \pi \xi_{j}\right)+\int_{-\infty}^{0} d x \frac{1-e^{-i x}}{i x}|x|^{-d_{1}-d_{2}} \exp \left(-i \pi \xi_{j}\right) \\
& =\int_{0}^{\infty} d x \frac{1-e^{-i x}}{i x} x^{-d_{1}-d_{2}} \exp \left(i \pi \xi_{j}\right)+\int_{0}^{\infty} d x \frac{1-e^{-i x}}{-i x} x^{-d_{1}-d_{2}} \exp \left(-i \pi \xi_{j}\right) \\
& =J \exp \left(i \pi \xi_{j}\right)+\bar{J} \exp \left(-i \pi \xi_{j}\right)
\end{aligned}
$$

where $\overline{(\cdot)}$ denotes complex conjugation and

$$
\begin{aligned}
J & =\int_{0}^{\infty} d x \frac{1-e^{-i x}}{i x^{1+d_{1}+d_{2}}}=\int_{0}^{\infty} d x \frac{1-\cos x+i \sin x}{i x^{1+d_{1}+d_{2}}} \\
& =-i \int_{0}^{\infty} d x \frac{1-\cos x}{x^{1+d_{1}+d_{2}}}+\int_{0}^{\infty} d x \frac{\sin x}{x^{1+d_{1}+d_{2}}} \\
& =\frac{\pi}{2 \Gamma\left(1+d_{1}+d_{2}\right)}\left[-i \csc \frac{\pi}{2}\left(d_{1}+d_{2}\right)+\sec \frac{\pi}{2}\left(d_{1}+d_{2}\right)\right] \\
& =\frac{\pi}{\Gamma\left(1+d_{1}+d_{2}\right)} \frac{-i \exp \left(i \frac{\pi}{2}\left(d_{1}+d_{2}\right)\right)}{\sin \pi\left(d_{1}+d_{2}\right)} .
\end{aligned}
$$

Using (A-12) in (A-11),

$$
\begin{aligned}
C\left(\xi_{j}\right) & =\frac{\pi}{\Gamma\left(1+d_{1}+d_{2}\right)} \frac{-i\left[\exp \left(i \pi\left(\frac{d_{1}+d_{2}}{2}+\xi_{j}\right)\right)-\exp \left(-i \pi\left(\frac{d_{1}+d_{2}}{2}+\xi_{j}\right)\right)\right]}{\sin \pi\left(d_{1}+d_{2}\right)} \\
& =\frac{2 \pi}{\Gamma\left(1+d_{1}+d_{2}\right)} \frac{\sin \pi\left(\frac{d_{1}+d_{2}}{2}+\xi_{j}\right)}{\sin \pi\left(d_{1}+d_{2}\right)}
\end{aligned}
$$

and

$$
\begin{aligned}
E \int_{0}^{1} X_{1} d X_{2} & =\frac{\sigma_{12}}{2 \pi} \frac{1}{1+d_{1}+d_{2}} \sum_{j=1}^{4} \theta_{j} C\left(\xi_{j}\right) \\
& =\frac{\sigma_{12}}{1+d_{1}+d_{2}} \frac{1}{\Gamma\left(1+d_{1}+d_{2}\right)} \frac{1}{\sin \pi\left(d_{1}+d_{2}\right)} \sum_{j=1}^{4} \theta_{j} \sin \pi\left(\frac{d_{1}+d_{2}}{2}+\xi_{j}\right) \\
& =\frac{\sigma_{12}}{1+d_{1}+d_{2}} \frac{\Gamma\left(d_{1}+d_{2}\right)}{\Gamma\left(1+d_{1}+d_{2}\right)} \frac{\Gamma\left(1-d_{1}-d_{2}\right)}{\pi} \sum_{j=1}^{4} \theta_{j} \sin \pi\left(\frac{d_{1}+d_{2}}{2}+\xi_{j}\right)
\end{aligned}
$$




$$
\begin{aligned}
& =\frac{\sigma_{12}}{1+d_{1}+d_{2}} \frac{\Gamma\left(1-d_{1}-d_{2}\right)}{\left(d_{1}+d_{2}\right)} \frac{1}{\pi} \sum_{j=1}^{4} \theta_{j} \sin \pi\left(\frac{d_{1}+d_{2}}{2}+\xi_{j}\right) \\
& =\frac{\sigma_{12}}{1+d_{1}+d_{2}} \frac{\Gamma\left(1-d_{1}-d_{2}\right)}{\left(d_{1}+d_{2}\right)} Q_{12}
\end{aligned}
$$

where

$$
Q_{12}=\frac{1}{\pi} \sum_{j=1}^{4} \theta_{j} \sin \pi\left(\frac{d_{1}+d_{2}}{2}+\xi_{j}\right)
$$

and direct substitution yields (4.3).

\section{A.6 Proof of Corollary 4.1}

Note that $E\left(X_{1}(1) X_{2}(1)\right)$ is given by setting $\delta=1$ in (3.3). We show that this formula matches the sum of (4.2) and the complementary expression having 1 and 2 interchanged. First note, using the relations

$$
\Gamma(1+x)=x \Gamma(x), \quad \Gamma(x) \Gamma(1-x)=\frac{\pi}{\sin \pi x},
$$

that

$$
\begin{aligned}
\frac{1}{\Gamma\left(2+d_{1}+d_{2}\right)} & =\frac{1}{1+d_{1}+d_{2}} \frac{1}{\Gamma\left(1+d_{1}+d_{2}\right)} \\
& =\frac{1}{1+d_{1}+d_{2}} \frac{\Gamma\left(1-d_{1}-d_{2}\right)}{d_{1}+d_{2}} \frac{1}{\Gamma\left(d_{1}+d_{2}\right) \Gamma\left(1-d_{1}-d_{2}\right)} \\
& =\frac{1}{1+d_{1}+d_{2}} \frac{\Gamma\left(1-d_{1}-d_{2}\right)}{d_{1}+d_{2}} \frac{\sin \pi\left(d_{1}+d_{2}\right)}{\pi}
\end{aligned}
$$

Next, from (4.3), using that fact that

$$
\kappa_{1} \kappa_{2}+\left(1-\kappa_{1}\right)\left(1-\kappa_{2}\right)=1-\kappa_{1}\left(1-\kappa_{2}\right)-\left(1-\kappa_{1}\right) \kappa_{2}
$$

and also the trigonometric identities

$$
\begin{aligned}
\sin \alpha+\sin \beta & =2 \sin \frac{\alpha+\beta}{2} \cos \frac{\alpha-\beta}{2} \\
\cos \alpha-\cos \beta & =-2 \sin \frac{\alpha+\beta}{2} \sin \frac{\alpha-\beta}{2} \\
\sin \alpha & =2 \sin \frac{\alpha}{2} \cos \frac{\alpha}{2},
\end{aligned}
$$

we complete the proof by noting

$$
\begin{aligned}
& Q_{12}+Q_{21}= \frac{1}{\pi}\left[\left(\kappa_{1} \kappa_{2}+\left(1-\kappa_{1}\right)\left(1-\kappa_{2}\right)\right)\left(\sin \pi\left(d_{2}+a_{1}-a_{2}\right)+\sin \pi\left(d_{1}+a_{2}-a_{1}\right)\right)\right. \\
&\left.+\left(\kappa_{1}\left(1-\kappa_{2}\right)+\left(1-\kappa_{1}\right) \kappa_{2}\right)\left(\sin \pi\left(a_{1}+a_{2}\right)+\sin \pi\left(d_{1}+d_{2}-a_{1}-a_{2}\right)\right)\right] \\
&=\frac{2}{\pi} \sin \pi \frac{d_{1}+d_{2}}{2}\left[\cos \pi\left(a_{1}-a_{2}-\frac{d_{1}-d_{2}}{2}\right)\right. \quad\left(\kappa_{1}\left(1-\kappa_{2}\right)+\left(1-\kappa_{1}\right) \kappa_{2}\right) \\
&\left.\quad \times\left(\cos \pi\left(a_{1}-a_{2}-\frac{d_{1}-d_{2}}{2}\right)-\cos \pi\left(a_{1}+a_{2}-\frac{d_{1}+d_{2}}{2}\right)\right)\right] \\
&=\frac{1}{\pi} \frac{\sin \pi\left(d_{1}+d_{2}\right)}{\cos \pi \frac{d_{1}+d_{2}}{2}}\left[\cos \pi\left(a_{1}-a_{2}-\frac{d_{1}-d_{2}}{2}\right)\right.
\end{aligned}
$$




$$
\left.-2\left(\kappa_{1}\left(1-\kappa_{2}\right)+\left(1-\kappa_{1}\right) \kappa_{2}\right) \sin \pi\left(a_{1}-\frac{d_{1}}{2}\right) \sin \pi\left(a_{2}-\frac{d_{2}}{2}\right)\right]
$$

\section{A.7 Proof of Theorem 4.1}

Following Chan and Terrin (1995, page 1674) define

$$
B_{n}(\mu, \lambda)=1_{[-n \pi, n \pi]^{2}}(\mu, \lambda) \frac{1}{n} \sum_{t=1}^{n-1} \frac{\exp (i(t+1) \mu / n)-\exp (i \mu / n)}{n(\exp (i \mu / n)-1)} \exp \left(\frac{i(t+1) \lambda}{n}\right)
$$

and

$$
B(\mu, \lambda)=\int_{0}^{1} \exp (i r \lambda) \frac{\exp (i r \mu)-1}{i \mu} d r .
$$

We prove, analogous to (3.14) of Chan and Terrin (1995), that

$$
\int_{-\infty}^{\infty} \int_{-\infty}^{\infty}\left|B_{n}(\mu, \lambda) n^{-d_{1}-d_{2}} g_{1}\left(e^{-i \mu / n}\right) g_{2}\left(e^{-i \lambda / n}\right)-B(\mu, \lambda) \tilde{g}_{1}(\mu) \tilde{g}_{2}(\lambda)\right|^{2} d \mu d \lambda \rightarrow 0
$$

as $n \rightarrow \infty$. Note that

$$
\begin{gathered}
\int_{-\infty}^{\infty} \int_{-\infty}^{\infty}\left|B_{n}(\mu, \lambda) n^{-d_{1}-d_{2}} g_{1}\left(e^{-i \mu / n}\right) g_{2}\left(e^{-i \lambda / n}\right)-B(\mu, \lambda) \tilde{g}_{1}(\mu) \tilde{g}_{2}(\lambda)\right|^{2} d \mu d \lambda \\
\leq K_{1}^{2} K_{2}^{2} \int_{-\infty}^{\infty} \int_{-\infty}^{\infty}\left|B_{n}(\mu, \lambda)-B(\mu, \lambda)\right|^{2}|\mu|^{-2 d_{1}}|\lambda|^{-2 d_{2}} d \mu d \lambda \\
\quad+\int_{-\infty}^{\infty} \int_{-\infty}^{\infty}\left|B_{n}(\mu, \lambda)\right|^{2}\left|\Phi_{n}(\lambda, \mu)\right|^{2}|\mu|^{-2 d_{1}}|\lambda|^{-2 d_{2}} d \mu d \lambda
\end{gathered}
$$

where $\tilde{g}_{i}(\lambda)=\tilde{g}\left(\lambda ; d_{i}, \kappa_{i}, a_{i}\right), g_{i}(L)=g\left(L ; d_{i}, \kappa_{i}, a_{i}\right)$ and $K_{i}=K\left(d_{i}, \kappa_{i}, a_{i}\right)$, for $i=1,2$, and

$$
\Phi_{n}(\lambda, \mu)=1_{[-n \pi, n \pi]^{2}}(\mu, \lambda)\left[|\mu|^{d_{1}}|\lambda|^{d_{2}}\left(n^{-d_{1}-d_{2}} g_{1}\left(e^{-i \mu / n}\right) g_{2}\left(e^{-i \lambda / n}\right)-\tilde{g}_{1}(\mu) \tilde{g}_{2}(\lambda)\right)\right] .
$$

The factor $1_{[-n \pi, n \pi]^{2}}(\mu, \lambda)$ can be optionally included in (A-14) since it does not change the value of the integral in (A-13).

It is required to show that both the terms on the majorant of (A-13) converge to 0 . It is easy to see that $B_{n}(\mu, \lambda)-B(\mu, \lambda) \rightarrow 0$ pointwise in $\mathbb{R}^{2}$, so in respect of the first term it suffices to show that

$$
\int_{-\infty}^{\infty} \int_{-\infty}^{\infty}\left|B_{n}(\mu, \lambda)\right|^{2}|\mu|^{-d_{1}}|\lambda|^{-d_{2}} d \mu d \lambda<\infty
$$

uniformly in $n$.

At points inside $[-\pi n, \pi n] \times[-\pi n, \pi n]$,

$$
\begin{aligned}
\left|B_{n}(\mu, \lambda)\right|^{2}= & \sum_{k=1}^{n-1} \frac{1}{n} \frac{\exp (i \mu(k+1) / n)-\exp (i \mu / n)}{n(\exp (i \mu / n)-1)} \exp (i \lambda(k+1) / n) \\
& \quad \times \sum_{j=1}^{n-1} \frac{1}{n} \frac{\exp (-i \mu(j+1) / n)-\exp (-i \mu / n)}{n(\exp (-i \mu / n)-1)} \exp (-i \lambda(j+1) / n) \\
= & V_{n}(\mu)+S_{n}(\mu, \lambda)+\bar{S}_{n}(\mu, \lambda)
\end{aligned}
$$

where

$$
V_{n}(\mu)=\sum_{k=1}^{n-1} \frac{1}{n^{2}} \frac{|\exp (i \mu(k+1) / n)-\exp (i \mu / n)|^{2}}{n^{2}|(\exp (i \mu / n)-1)|^{2}}=\frac{1}{n^{4}} \sum_{k=1}^{n-1}\left[\frac{\sin (\mu k /(2 n))}{\sin (\mu /(2 n))}\right]^{2},
$$




$$
\begin{aligned}
S_{n}= & \sum_{k=2}^{n-1} \sum_{j=1}^{k-1} \frac{1}{n^{2}} \frac{[\exp (i \mu(k+1) / n)-\exp (i \mu / n)][\exp (-i \mu(j+1) / n)-\exp (-i \mu / n)]}{n^{2}(\exp (i \mu / n)-1)(\exp (-i \mu / n)-1)} \\
& \times \exp (i \lambda(k-j) / n) \\
= & \sum_{k=2}^{n-1} \sum_{j=1}^{k-1} \frac{1}{n^{4}} \frac{\exp (i \mu(k-j) / n)-\exp (-i \mu j / n)-\exp (i \mu k / n)+1}{(\exp (i \mu / n)-1)(\exp (-i \mu / n)-1)} \exp (i \lambda(k-j) / n)
\end{aligned}
$$

and $\bar{S}_{n}$ is the complex conjugate of $S_{n}$. Consider the last two terms in (A-15). Note that

$$
\begin{aligned}
& \int_{-\pi n}^{\pi n} \int_{-\pi n}^{\pi n}\left(S_{n}+\bar{S}_{n}\right)|\mu|^{-2 d_{1}}|\lambda|^{-2 d_{2}} d \mu d \lambda \\
& =\frac{1}{n^{2}} \sum_{k=2}^{n-1} \sum_{j=1}^{k-1}\left[\int_{-\pi n}^{\pi n} \exp (i \lambda(k-j) / n) \frac{d \lambda}{|\lambda|^{2 d_{2}}}\right. \\
& \times \int_{-\pi n}^{\pi n} \frac{\exp (i \mu(k-j) / n)-\exp (-i \mu j / n)-\exp (i \mu k / n)+1}{n^{2}(\exp (i \mu / n)-1)(\exp (-i \mu / n)-1)} \frac{d \mu}{|\mu|^{2 d_{1}}} \\
& +\int_{-\pi n}^{\pi n} \exp (-i \lambda(k-j) / n) \frac{d \lambda}{|\lambda|^{2 d_{2}}} \\
& \left.\times \int_{-\pi n}^{\pi n} \frac{\exp (-i \mu(k-j) / n)-\exp (i \mu j / n)-\exp (-i \mu k / n)+1}{n^{2}(\exp (i \mu / n)-1)(\exp (-i \mu / n)-1)} \frac{d \mu}{|\mu|^{2 d_{1}}}\right] \\
& =\frac{1}{n^{2}} \sum_{k=2}^{n-1} \sum_{j=1}^{k-1}\left[\int_{-\pi n}^{\pi n} \exp (i \lambda(k-j) / n) \frac{d \lambda}{|\lambda|^{2 d_{2}}}\right. \\
& \times \int_{-\pi n}^{\pi n} \frac{\exp (i \mu(k-j) / n)-\exp (-i \mu j / n)-\exp (i \mu k / n)+1}{n^{2}(\exp (i \mu / n)-1)(\exp (-i \mu / n)-1)} \frac{d \mu}{|\mu|^{2 d_{1}}} \\
& +\int_{-\pi n}^{\pi n} \exp (i \lambda(k-j) / n) \frac{d \lambda}{|\lambda|^{2 d_{2}}} \\
& \left.\times \int_{-\pi n}^{\pi n} \frac{\exp (-i \mu(k-j) / n)-\exp (i \mu j / n)-\exp (-i \mu k / n)+1}{n^{2}(\exp (i \mu / n)-1)(\exp (-i \mu / n)-1)} \frac{d \mu}{|\mu|^{2 d_{1}}}\right] \\
& =\frac{1}{n^{2}} \sum_{k=2}^{n-1} \sum_{j=1}^{k-1} \int_{-\pi n}^{\pi n} \cos \frac{\lambda(k-j)}{n} \frac{d \lambda}{|\lambda|^{2 d_{2}}} \\
& \times \int_{-\pi n}^{\pi n} \frac{2(1+\cos (\mu(k-j) / n)-\cos (\mu j / n)-\cos (\mu k / n))}{2(1-\cos (\mu / n))} \frac{d \mu}{|\mu|^{2 d_{1}}} \\
& =\frac{4}{n^{2}} \sum_{k=2}^{n-1} \sum_{j=1}^{k-1} \int_{0}^{\pi n} \cos \frac{\lambda(k-j)}{n} \frac{d \lambda}{\lambda^{2 d_{2}}} \\
& \times \int_{0}^{\pi n} \frac{\sin ^{2}(\mu j /(2 n))+\sin ^{2}(\mu k /(2 n))-\sin ^{2}(\mu(k-j) /(2 n))}{n^{2} \sin ^{2}(\mu /(2 n))} \frac{d \mu}{\mu^{2 d_{1}}}
\end{aligned}
$$

where in the second equality we did a change of variable from $\lambda$ to $-\lambda$ and in the third we collected terms and cancelled odd components.

Consider first the integral with respect to $\lambda$.

$$
\int_{0}^{\pi n} \cos \frac{\lambda(k-j)}{n} \frac{d \lambda}{\lambda^{2 d_{2}}}=\int_{0}^{1} \cos \frac{\lambda(k-j)}{n} \frac{d \lambda}{\lambda^{2 d_{2}}}+\int_{1}^{\pi n} \cos \frac{\lambda(k-j)}{n} \frac{d \lambda}{\lambda^{2 d_{2}}}
$$




$$
\begin{aligned}
& \leq \int_{0}^{1} \frac{d \lambda}{\lambda^{2 d_{2}}}+\int_{1}^{\pi n} \cos \frac{\lambda(k-j)}{n} d \lambda \\
& =\frac{1}{1-2 d_{2}}-\frac{\sin (k-j) / n}{(k-j) / n} \\
& \leq \frac{1}{1-2 d_{2}}-1
\end{aligned}
$$

using $\left|\frac{\sin x}{x}\right| \leq 1$. Next consider the integral with respect to $\mu$. Using (A-2) and (A-3) we have

$$
\begin{aligned}
& \int_{0}^{\pi n} \frac{\sin ^{2}(\mu j /(2 n))+\sin ^{2}(\mu k /(2 n))-\sin ^{2}(\mu(k-j) /(2 n))}{n^{2} \sin ^{2}(\mu /(2 n))} \frac{d \mu}{\mu^{2 d_{1}}} \\
& \leq \int_{0}^{1} \frac{\sin ^{2}(\mu j /(2 n))+\sin ^{2}(\mu k /(2 n))+\sin ^{2}(\mu(k-j) /(2 n))}{n^{2} \sin ^{2}(\mu /(2 n))} \frac{d \mu}{\mu^{2 d_{1}}} \quad+\int_{1}^{\pi n} \frac{\sin ^{2}(\mu j /(2 n))+\sin ^{2}(\mu k /(2 n))+\sin ^{2}(\mu(k-j) /(2 n))}{n^{2} \sin ^{2}(\mu /(2 n))} \frac{d \mu}{\mu^{2 d_{1}}} \\
& \leq \int_{0}^{1} \frac{1}{n^{2}}\left(j^{2}+k^{2}+(k-j)^{2}\right) \frac{d \mu}{\mu^{2 d_{1}}} \\
& \quad+\int_{0}^{\pi n} \frac{\sin ^{2}(\mu j /(2 n))+\sin ^{2}(\mu k /(2 n))+\sin ^{2}(\mu(k-j) /(2 n))}{n^{2} \sin ^{2}(\mu /(2 n))} d \mu \\
& \leq \int_{0}^{1} 6 \frac{d \mu}{\mu^{2 d_{1}}}+\frac{2 n}{n^{2}} \pi(j+k+(k-j)) \\
& \leq \frac{6}{1-2 d_{1}}+4 \pi .
\end{aligned}
$$

Substituting these bounds in (A-16) yields

$$
\int_{-\pi n}^{\pi n} \int_{-\pi n}^{\pi n}\left(S_{n}+\bar{S}_{n}\right)|\mu|^{-2 d_{1}}|\lambda|^{-2 d_{2}} d \mu d \lambda \leq 4\left(\frac{1}{1-2 d_{2}}+1\right)\left(\frac{6}{1-2 d_{1}}+4 \pi\right) .
$$

Next, considering the first term in (A-15), note that

$$
\begin{aligned}
\int_{-\pi n}^{\pi n} \int_{-\pi n}^{\pi n} & V_{n}(\mu)|\mu|^{-2 d_{1}}|\lambda|^{-2 d_{2}} d \mu d \lambda \\
& =\frac{1}{n} \int_{-\pi n}^{\pi n}|\lambda|^{-2 d_{2}} d \lambda \frac{1}{n^{3}} \sum_{k=1}^{n-1} \int_{-\pi n}^{\pi n} \frac{\sin ^{2}(\mu k /(2 n))}{\sin ^{2}(\mu /(2 n))}|\mu|^{-2 d_{1}} d \mu \\
& =\frac{4}{n} \int_{0}^{\pi n} \lambda^{-2 d_{2}} d \lambda \frac{1}{n^{3}} \sum_{k=1}^{n-1} \int_{0}^{\pi n} \frac{\sin ^{2}(\mu k /(2 n))}{\sin ^{2}(\mu /(2 n))} \mu^{-2 d_{1}} d \mu \\
& =\frac{4 \pi^{1-2 d_{2}}}{1-2 d_{2}} n^{-2 d_{2}} \frac{1}{n^{3}} \sum_{k=1}^{n-1}\left(\int_{0}^{1} \frac{\sin ^{2}(\mu k /(2 n))}{\sin ^{2}(\mu /(2 n))} \mu^{-2 d_{1}} d \mu+\int_{1}^{\pi n} \frac{\sin ^{2}(\mu k /(2 n))}{\sin ^{2}(\mu /(2 n))} \mu^{-2 d_{1}} d \mu\right) \\
& \leq \frac{4 \pi^{1-2 d_{2}}}{1-2 d_{2}} n^{-2 d_{2}} \frac{1}{n^{3}} \sum_{k=1}^{n-1}\left(\int_{0}^{1} k^{2} \mu^{-2 d_{1}} d \mu+\int_{1}^{\pi n} \frac{\sin ^{2}(\mu k /(2 n))}{\sin ^{2}(\mu /(2 n))} d \mu\right) \\
& \leq \frac{4 \pi^{1-2 d_{2}}}{1-2 d_{2}} n^{-2 d_{2}} \frac{1}{n^{3}} \sum_{k=1}^{n-1}\left(\frac{n^{2}}{1-2 d_{1}}+\int_{0}^{\pi n} \frac{\sin ^{2}(\mu k /(2 n))}{\sin ^{2}(\mu /(2 n))} d \mu\right) \\
& =\frac{4 \pi^{1-2 d_{2}}}{1-2 d_{2}} n^{-2 d_{2}} \frac{1}{n^{3}} \sum_{k=1}^{n-1}\left(\frac{n^{2}}{1-2 d_{1}}+2 n \frac{\pi k}{2}\right)
\end{aligned}
$$




$$
\begin{aligned}
& \leq \frac{4 \pi^{1-2 d_{2}}}{1-2 d_{2}} n^{-2 d_{2}} \frac{1}{n^{3}} \sum_{k=1}^{n-1}\left(\frac{n^{2}}{1-2 d_{1}}+n^{2} \pi\right) \\
& \leq \frac{4 \pi^{1-2 d_{2}}}{1-2 d_{2}} n^{-2 d_{2}}\left(\frac{1}{1-2 d_{1}}+\pi\right) \\
& \leq \frac{4 \pi^{1-2 d_{2}}}{1-2 d_{2}}\left(\frac{1}{1-2 d_{1}}+\pi\right)<\infty .
\end{aligned}
$$

Observe that this term vanishes for $d_{2}>0$, although not for $d_{2}=0$.

The same argument can be invoked again to show that the second majorant term of (A-13) vanishes, provided $\Phi_{n}(\lambda, \mu)$ is bounded uniformly in $n$, and converges to 0 pointwise in $\mathbb{R}^{2}$. To show this, recall that

$$
\tilde{g}_{1}(\mu) \tilde{g}_{2}(\lambda)=|\mu|^{-d_{1}}|\lambda|^{-d_{2}} \Upsilon\left(\mu ; d_{1}, \kappa_{1}, a_{1}\right) \Upsilon\left(\lambda ; d_{2}, \kappa_{2}, a_{2}\right)
$$

where

$$
\begin{aligned}
& \Upsilon\left(\mu ; d_{1}, \kappa_{1}, a_{1}\right) \Upsilon\left(\lambda ; d_{2}, \kappa_{2}, a_{2}\right) \\
& =\kappa_{1} \kappa_{2} e^{-i \pi\left[\left(d_{1} / 2-a_{1}\right) \operatorname{sgn}(\mu)+\left(d_{2} / 2-a_{2}\right) \operatorname{sgn}(\lambda)\right]} \\
& \quad+\left(1-\kappa_{1}\right)\left(1-\kappa_{2}\right) e^{i \pi\left[\left(d_{1} / 2-a_{1}\right) \operatorname{sgn}(\mu)+\left(d_{2} / 2-a_{2}\right) \operatorname{sgn}(\lambda)\right]} \\
& \quad+\kappa_{1}\left(1-\kappa_{2}\right) e^{-i \pi\left[\left(d_{1} / 2-a_{1}\right) \operatorname{sgn}(\mu)-\left(d_{2} / 2-a_{2}\right) \operatorname{sgn}(\lambda)\right]} \\
& \quad+\left(1-\kappa_{1}\right) \kappa_{2} e^{i \pi\left[\left(d_{1} / 2-a_{1}\right) \operatorname{sgn}(\mu)-\left(d_{2} / 2-a_{2}\right) \operatorname{sgn}(\lambda)\right]} .
\end{aligned}
$$

From (2.12) the expression $n^{-d_{1}-d_{2}} g\left(e^{-i \mu / n} ; d_{1}, \kappa_{1}, a_{1}\right) g\left(e^{-i \lambda / n} ; d_{2}, \kappa_{2}, a_{2}\right)$ can be decomposed similarly, into terms with coefficients $\kappa_{1} \kappa_{2},\left(1-\kappa_{1}\right)\left(1-\kappa_{2}\right), \kappa_{1}\left(1-\kappa_{2}\right)$ and $\left(1-\kappa_{1}\right) \kappa_{2}$. Pairing up these matching terms, $\Phi_{n}(\lambda, \mu)$ can be therefore be decomposed (defining functions $\phi_{k n}$, $k=1, \ldots, 4$ in the obvious manner) as

$$
\begin{aligned}
\Phi_{n}(\lambda, \mu)=\kappa_{1} \kappa_{2} \phi_{1 n}(\mu, \lambda) & +\left(1-\kappa_{1}\right)\left(1-\kappa_{2}\right) \phi_{2 n}(\mu, \lambda) \\
& +\kappa_{1}\left(1-\kappa_{2}\right) \phi_{3 n}(\mu, \lambda)+\left(1-\kappa_{1}\right) \kappa_{2} \phi_{4 n}(\mu, \lambda) .
\end{aligned}
$$

We consider the term $\phi_{1 n}$ as representative. This takes the form

$$
\begin{gathered}
\phi_{1 n}(\mu, \lambda)=1_{[-n \pi, n \pi]^{2}}(\mu, \lambda)|\mu|^{d_{1}}|\lambda|^{d_{2}} \\
\times\left[n^{-d_{1}-d_{2}}\left(1-e^{-i \mu / n}\right)^{a_{1}-d_{1}}\left(1-e^{i \mu / n}\right)^{-a_{1}}\left(1-e^{-i \lambda / n}\right)^{a_{2}-d_{2}}\left(1-e^{i \lambda / n}\right)^{-a_{2}}\right. \\
\left.-e^{-i \pi\left[\left(d_{1} / 2-a_{1}\right) \operatorname{sgn}(\mu)+\left(d_{2} / 2-a_{2}\right) \operatorname{sgn}(\lambda)\right]}\right] .
\end{gathered}
$$

Note that

$$
\begin{aligned}
n^{s}\left(1-e^{-i \mu / n}\right)^{s} & =\left(i \mu+O\left(n^{-1}\right)\right)^{s} \\
& =|\mu|^{s}\left(e^{i \pi \operatorname{sgn}(\mu) / 2}+O\left(n^{-1}\right)\right)^{s}
\end{aligned}
$$

and hence

$$
\begin{gathered}
\phi_{1 n}(\mu, \lambda)=1_{[-n \pi, n \pi]^{2}}(\mu, \lambda)\left[\left(\left(e^{i \pi \operatorname{sgn}(\mu) / 2}+O\left(n^{-1}\right)\right)^{a_{1}-d_{1}}\left(e^{-i \pi \operatorname{sgn}(\mu) / 2}+O\left(n^{-1}\right)\right)^{-a_{1}}\right)\right. \\
\left.\times\left(e^{i \pi \operatorname{sgn}(\lambda) / 2}+O\left(n^{-1}\right)\right)^{a_{2}-d_{2}}\left(e^{-i \pi \operatorname{sgn}(\lambda) / 2}+O\left(n^{-1}\right)\right)^{-a_{2}}\right) \\
\left.-e^{-i \pi\left[\left(d_{1} / 2-a_{1}\right) \operatorname{sgn}(\mu)+\left(d_{2} / 2-a_{2}\right) \operatorname{sgn}(\lambda)\right]}\right] .
\end{gathered}
$$

It can be verified that $\phi_{1 n}(\mu, \lambda)$ is absolutely bounded everywhere in $\mathbb{R}^{2}$, and $\phi_{1 n}(\mu, \lambda)=O\left(n^{-1}\right)$ follows similarly to (A-6). Precisely parallel arguments apply to the terms $\phi_{k n}(\mu, \lambda)$ for $k=2,3,4$. This completes the proof. 


\section{A.8 Proof of Lemma 5.1}

We need to compute the integral

$$
G(d)=\int_{0}^{\infty}\left[(x+1)^{d}-x^{d}\right]^{2} d x
$$

for $-1 / 2<d<1 / 2$. Denote the integrand by $f(x)$. Note that for $d=0$ the integral is trivially zero. For $0<d<1 / 2$ we have $\lim _{x \rightarrow \pm \infty}|f(x)|=0, \lim _{x \rightarrow 0}|f(x)|=1$, and the function is integrable, for both positive and negative $x$. For $-1 / 2<d<0$, we have $\lim _{x \rightarrow \pm \infty}|f(x)|=0$, and $f(x)$ has a singularity at $x=0$ with $\lim _{x \rightarrow 0}\left|f(x) x^{-2 d}\right|=1$. Hence, is it integrable on the positive half-line. For $x<0$ it also has a singularity at $x=-1$ with $\lim _{x \rightarrow-1}\left|f(x)(x+1)^{-2 d}\right|=1$, and, therefore, is also integrable on the negative half-line.

Thus, consider the auxiliary integral

$$
G^{*}(d)=\int_{-\infty}^{\infty} f(x) d x
$$

It is easy to see that for $d \neq 0$ this integral equals zero:

$$
\begin{aligned}
G^{*}(d) & =\int_{-\infty}^{\infty}\left[(x+1)^{d}-x^{d}\right]^{2} d x \\
& =\int_{-\infty}^{\infty}\left[(-y-1+1)^{d}-(-y-1)^{d}\right]^{2} d y \\
& =(-1)^{2 d} \int_{-\infty}^{\infty}\left[y^{d}-(y+1)^{d}\right]^{2} d y \\
& =e^{2 i \pi d} G^{*}(d) .
\end{aligned}
$$

Next, divide the range of integration into $(-\infty,-1),(-1,0)$, and $(0, \infty)$. For the first interval change of variables $\tilde{x}=-x-1$ gives

$$
\begin{aligned}
\int_{-\infty}^{-1} f(x) d x & =\int_{-\infty}^{-1}\left[(x+1)^{d}-x^{d}\right]^{2} d x \\
& =\int_{0}^{\infty}\left[(-\tilde{x})^{d}-(-\tilde{x}-1)^{d}\right]^{2} d \tilde{x} \\
& =(-1)^{2 d} \int_{0}^{\infty}\left[\tilde{x}^{d}-(\tilde{x}+1)^{d}\right]^{2} d \tilde{x} \\
& =e^{2 i \pi d} G(d) .
\end{aligned}
$$

For the second interval we have

$$
\begin{aligned}
\int_{-1}^{0} f(x) d x & =\int_{-1}^{0}\left[(x+1)^{d}-x^{d}\right]^{2} d x \\
& =\int_{-1}^{0}\left[(x+1)^{2 d}+x^{2 d}-2(x+1)^{d} x^{d}\right] d x \\
& =\frac{1}{2 d+1}(1-0)+\frac{1}{2 d+1}\left(0-(-1)^{2 d+1}\right)-2(-1)^{d} \int_{0}^{1} \bar{x}^{d}(1-\bar{x})^{d} d \bar{x} \\
& =\frac{1+e^{2 i \pi d}}{2 d+1}-2 e^{i \pi d} B(d+1, d+1)
\end{aligned}
$$


where in the last integral we used change of variables $\bar{x}=x+1$. The integral over the third interval is simply $G(d)$. Adding the integrals over these three intervals we obtain

$$
e^{2 i \pi d} G(d)+\frac{1+e^{2 i \pi d}}{2 d+1}-2 e^{i \pi d} B(d+1, d+1)+G(d)=0,
$$

or,

$$
\begin{aligned}
G(d) & =\frac{1}{1+e^{2 i \pi d}}\left(-\frac{1+e^{2 i \pi d}}{2 d+1}+2 e^{i \pi d} B(d+1, d+1)\right) \\
& =\frac{1}{2 d+1}[-1+(2 d+1) \sec (\pi d) B(d+1, d+1)] .
\end{aligned}
$$

Note that this expression formally captures the result $G(0)=0$. Further, using the functional relationships

$$
B(x, y)=\frac{\Gamma(x) \Gamma(y)}{\Gamma(x+y)}, \quad \Gamma(1-x) \Gamma(x)=\frac{\pi}{\sin x}, \quad \Gamma(x+1)=x \Gamma(x),
$$

we can express the integral equivalently as

$$
G(d)=\frac{1}{2 d+1}\left(\frac{[\Gamma(d+1)]^{2} \sec (\pi d)}{\Gamma(2 d+1)}-1\right)
$$

or

$$
G(d)=\frac{1}{2 d+1}\left(\frac{\Gamma(1-2 d) \Gamma(1+d)}{\Gamma(1-d)}-1\right) .
$$

\section{A.9 Proof of Proposition 5.1}

By (5.3), and the orthogonality of Brownian motion, we may write

$$
\begin{gathered}
E[X(r+\delta)-X(r)]^{2} \\
=\sigma^{2} \int_{r}^{r+\delta}\left(\kappa^{2}(r+\delta-s)^{2 d}+(1-\kappa)^{2}(s-r)^{2 d}+2 \kappa(1-\kappa)(r+\delta-s)^{d}(s-r)^{d}\right) d s \\
+\sigma^{2} \kappa^{2} \int_{-\infty}^{r}\left[(r+\delta-s)^{d}-(r-s)^{d}\right]^{2} d s \\
\quad+\sigma^{2}(1-\kappa)^{2} \int_{r+\delta}^{\infty}\left[(s-r-\delta)^{d}-(s-r)^{d}\right]^{2} d s \\
=\sigma^{2} \delta^{2 d+1}\left(\int_{0}^{1}\left(\kappa^{2}(1-s)^{2 d}+(1-\kappa)^{2} s^{2 d}+2 \kappa(1-\kappa)(1-s)^{d} s^{d}\right) d s\right. \\
\left.\quad+(1-2 \kappa(1-\kappa)) \int_{0}^{\infty}\left[(1+s)^{d}-s^{d}\right]^{2} d s\right) \\
=\delta^{2 d+1} \frac{\sigma^{2}}{2 d+1}\left((1-2 \kappa(1-\kappa)) \frac{\Gamma(1-2 d)}{\Gamma(1-d)}+2 \kappa(1-\kappa) \frac{\Gamma(d+1)^{2}}{\Gamma(2 d+1)}\right)
\end{gathered}
$$

and the proposition follows directly on rearrangement. Note that the second equality makes a change of variable, and the third one substitutes the complete beta function and from Lemma 5.1 . 


\section{A.10 Proof of Proposition 5.2}

Use the properties of the gamma function

$$
\Gamma(x) \Gamma(1-x)=\frac{\pi}{\sin \pi x}, \quad \Gamma(x+1)=x \Gamma(x)
$$

to do the following transformation:

$$
\begin{aligned}
\frac{\Gamma(1-2 d)}{\Gamma(1-d)} & =\frac{\pi}{\Gamma(2 d) \sin 2 \pi d} \frac{\Gamma(d) \sin \pi d}{\pi} \\
& =\frac{2 d}{\Gamma(2 d+1)} \frac{\Gamma(d+1)}{d} \frac{\sin \pi d}{2 \sin \pi d \cos \pi d} \\
& =\frac{\Gamma(d+1)}{\Gamma(2 d+1)} \frac{1}{\cos \pi d} .
\end{aligned}
$$

Substituting this equality yields

$$
\begin{aligned}
V & =\frac{\sigma^{2}}{(2 d+1) \Gamma(d+1)}\left[(1-2 \kappa(1-\kappa)) \frac{\Gamma(d+1)}{\Gamma(2 d+1)} \frac{1}{\cos \pi d}+2 \kappa(1-\kappa) \frac{\Gamma(d+1)}{\Gamma(2 d+1)}\right] \\
& =\frac{\sigma^{2}}{(2 d+1) \Gamma(d+1)} \frac{\Gamma(d+1)}{\Gamma(2 d+1)} \frac{1-2 \kappa(1-\kappa)+2 \kappa(1-\kappa) \cos \pi d}{\cos \pi d} \\
& =\sigma^{2} \frac{1-2 \kappa(1-\kappa)(1-\cos \pi d)}{\Gamma(2 d+2) \cos \pi d} \\
& =\sigma^{2} \frac{K(d, \kappa, 0)^{2}}{\Gamma(2 d+2) \cos \pi d} .
\end{aligned}
$$

\section{A.11 Proof of Theorem 5.1}

Write $x_{t}=\kappa x_{b t}+(1-\kappa) x_{f t}$ where

$$
x_{b t}=(1-L)^{-d} u_{t}, \quad x_{f t}=\left(1-L^{-1}\right)^{-d} u_{t}
$$

The result $X_{b n} \stackrel{d}{\rightarrow} X_{b}$, where $X_{b n}(r)=n^{-1 / 2-d} \sum_{t=1}^{[n r]} x_{b t}$ and $X_{b}$ is the process in (5.1), follows from Theorem 3.1 of Davidson and de Jong (2000) (henceforth, DDJ).

To deal with $X_{f n}$, the forward-looking increment process can be converted into a backwardlooking process by reversing the time ordering. Define $v_{n t}=u_{n-t+1}$ for $-\infty<t<\infty$, and let

$$
z_{n t}=x_{f, n-t+1}=(1-L)^{-d} v_{n t}
$$

Then,

$$
\begin{aligned}
X_{f n}(r) & =n^{-1 / 2-d} \sum_{t=1}^{[n r]} x_{f t} \\
& =n^{-1 / 2-d} \sum_{t=1}^{n} x_{f t}-n^{-1 / 2-d} \sum_{t=[n r]+1}^{n} x_{f t} \\
& =n^{-1 / 2-d} \sum_{t=1}^{n} z_{n t}-n^{-1 / 2-d} \sum_{t=1}^{n-[n r]} z_{n t} .
\end{aligned}
$$

Although $\left\{v_{n t}\right\}$ and $\left\{z_{n t}\right\}$ are technically arrays, note that they are stationary processes. Their distribution is invariant to $n$, and they satisfy the conditions of DDJ Assumption 1 for any choice 
of $n$. In effect, $X_{f n}$ is equivalent to a 'backward-looking' partial sum process in which the starting date shifts backwards in time as $n$ increases, with a fixed terminal date. However, the sequence of distributions defined by letting $n \rightarrow \infty$ is invariant to these shifts, and matches that of the conventional 'fixed start' process at each point. Hence, DDJ Theorerm 3.1 can be invoked to establish the joint weak convergence of each term in (A-17), and $X_{f n} \stackrel{d}{\rightarrow} X_{f}$ by the continuous mapping theorem. The limiting random variable for each $r$ takes the form.

$$
\begin{aligned}
X_{f}(r) & =\int_{0}^{1}(1-s)^{d} d B-\int_{0}^{1-r}(r-s)^{d} d B-\int_{-\infty}^{0}\left[(r-s)^{d}-(-s)^{d}\right] d B \\
& =\int_{0}^{1} s^{d} d B-\int_{r}^{1} s^{d} d B+\int_{r}^{\infty}\left[s^{d}-(s-r)^{d}\right] d B \\
& =\int_{0}^{r} s^{d} d B+\int_{r}^{\infty}\left[s^{d}-(s-r)^{d}\right] d B
\end{aligned}
$$

where the second and third equalities follows by changes of variables and rearrangement.

Finally, it further follows by the continuous mapping theorem that

$$
\begin{aligned}
& X_{n}=\kappa X_{b n}+(1-\kappa) X_{f n} \\
& \quad \stackrel{d}{\rightarrow} \kappa X_{b}+(1-\kappa) X_{f}=X
\end{aligned}
$$

Note that this argument depends on the $\left\{u_{t}\right\}$ process being reversible without altering the dependence structure. Thus, if the initial FCLT was established using the assumption that $u_{t}$ was a martingale difference, the second stage of the argument would fail. However, the theorem of DDJ specifies that $u_{t}$ is $L_{2}$-near-epoch dependent on a mixing process, and this characterization of dependence is preserved under time reversal.

\section{References}

Beran, J. (1994) Statistics for Long Memory Processes. New York: Chapman and Hall.

Brockwell, P. J. and R. A. Davis (1991) Time Series: Theory and Methods, 2nd edn. SpringerVerlag.

Chan, N. H. and N. Terrin (1995) Inference for unstable long-memory processes with applications to fractional unit root autoregressions. Annals of Statistics 25,5, 1662-1683.

Davidson, J. (1994) Stochastic Limit Theory. Oxford University Press

Davidson, J. and R. M. de Jong (2000) The functional central limit theorem and convergence to stochastic integrals II: fractionally integrated processes. Econometric Theory 16, 5, 643-666.

Duncan, T. E, Y. Hu, and B. Pasik-Duncan (2000) Stochastic calculus for fractional Brownian motion. I Theory. SIAM J. Control Optim. 38, 2, 582-612.

Flandrin, P. (1989) On the Spectrum of Fractional Brownian Motions, IEEE Transactions on Information Theory, 35, 1, 197-199.

Fox, R. and M. Taqqu (1987) Multiple stochastic integrals with dependent integrators. Journal of Multivariate Analysis 21, 105-127.

Gradshteyn and Ryzhik (2000) Tables of Integrals, Series, and Products (6th Edn), eds A. Jeffrey and D. Zwillinger. Academic Press.

Granger, C.W.J. and Roselyne Joyeux (1980) An introduction to long memory time series models and fractional differencing. Journal of Time Series Analysis 1, 1, 15-29.

Hosking, J. R. M. (1981) Fractional differencing. Biometrika 68,1, 165-76. 
Kim, C. S. and P. C. B. Phillips (2001) Fully modified estimation of fractional cointegration models, Working Paper at http://www.sceco.umontreal.ca/cesg/pdf_files/Kim-cesg_4m.pdf

Major, P. (1981) Multiple Wiener-Itô Integrals, with Applications to Limit Theorems. Lecture Notes in Mathematics 849, Berlin: Springer-Verlag.

Mandelbrot, B. B. and J. W. van Ness (1968) Fractional Brownian motions, fractional noises and applications. SIAM Review 10, 4, 422-437.

Marinucci, D. and P. M. Robinson (1999) Alternative forms of fractional Brownian motion. Journal of Statistical Inference and Planning 80, 111-122.

Marinucci, D. and P. M. Robinson (2000) Weak convergence of multivariate fractional processes. Stochastic Processes and their Applications 86, pp.103-120.

Marinucci, D. and P. M. Robinson (2001) Semiparametric fractional cointegration analysis. Journal of Econometrics 105, 225-247

Reed, Irving S., P. C. Lee, and T. K. Truong (1995) Spectral Representation of Fractional Brownian Motion in $n$ dimensions and its Properties, IEEE Transactions on Information Theory, 41, 5, 1439-1451.

Robinson, P. M. (1994a) Time series with strong dependence. In Advances in Econometrics, Econometric Society 6th World Congress. Vol. 1 (Sims, ed.) Cambridge University Press.

Robinson, P. M. (1994b), Semiparametric analysis of long memory time series, Annals of Statistics $22,1,515-539$.

Robinson, P. M. and D. Marinucci (2001) Narrow band analysis of nonstationary processes. Annals of Statistics 29, 947-986

Robinson, P. M. and D. Marinucci (2003) Semiparametric frequency domain analysis of fractional cointegration, in Time Series with Long Memory (ed. P. M. Robinson), Oxford University Press.

Taqqu, M. S. (1975) Weak convergence to fractional Brownian motion and to the Rosenblatt process. Z. Wahrsch. Verw. Gebiete, 31, 287-302. 